\title{
Bilinear Paraproducts Revisited
}

\author{
Árpad Bényi ${ }^{1}$, Diego Maldonado ${ }^{2}$, Andrea R. Nahmod ${ }^{3}$, and Rodolfo H. Torres ${ }^{* 4}$ \\ ${ }^{1}$ Department of Mathematics, Western Washington University, Bellingham, WA 98225-9063, USA \\ 2 Department of Mathematics, Kansas State University, Manhattan, KS 66506, USA \\ ${ }^{3}$ Department of Mathematics and Statistics, Lederle GRT, University of Massachusetts, Amherst, MA 01003, USA \\ ${ }^{4}$ Department of Mathematics, University of Kansas, 405 Snow Hall, 1460 Jayhawk Blvd, Lawrence, Kansas 66045-7523, \\ USA
}

Received 31 August 2007

Key words Singular integrals, maximal functions, Littlewood-Paley theory, multilinear operators, almost diagonal condition, wavelets, molecules, Sobolev spaces, Triebel-Lizorkin spaces MSC (2000) Primary 42B25, 42B20, 47G30. Secondary 42C15, 46E35, 35 S 99

Boundedness properties for bilinear paraproducts on several function spaces are presented. The methods are based on the realization of paraproducts as bilinear Calderón-Zygmund operators and the molecular characterization of function spaces. This provides a unified approach for the study of paraproducts, recovering some know results and establishing several new.

Copyright line will be provided by the publisher

\section{Introduction}

Starting with the work of Coifman and Meyer in the 70's (see for instance [8]) the multilinear approach to study product-like operators has been followed numerous times in the literature. In addition to their relevance in nonlinear partial differential equations, product-like operations are of interest in harmonic analysis in the study of linear and multilinear singular integrals. Some recent works involving estimates for multilinear singular integrals of relevance for this article include [1], [2], [7], [13] [14], [15], [16], [17], [19], [20], [21], [22], [24], [26], [28], [35]. This is only a partial list of the numerous research works in an area that continues to attract a lot of attention.

The name paraproduct has been used somewhat loosely in the literature to denote different types of operators which behave like (and often, as we shall see again in this article, better than) the product of functions. The paraproduct paradigm can be traced back to the contributions by H. Triebel [30], [31], as well as those by J. Petree [27], to the study of multiplication properties in Besov and Triebel-Lizorkin spaces. See [32] for further references. The term paraproduct, however, goes back to the seminal work of Bony in [5], where the words paramultiplication and paradifferential operator were introduced and some of its uses in non-linear partial differential equations were established. In general terms, a paraproduct consists of sums or integrals that superimpose various time-frequency components of the functions on which it acts. In particular, we will reexamine the bilinear operators (broadly considered in the literature) of the form

$$
T(f, g)(x)=\sum_{Q}|Q|^{-1 / 2}\left\langle f, \phi_{Q}^{1}\right\rangle\left\langle g, \phi_{Q}^{2}\right\rangle \phi_{Q}^{3}(x)
$$

where the sum runs over all dyadic cubes in $\mathbf{R}^{n}$ and the functions $\phi_{Q}^{i}, i=1,2,3$, are families of molecules (see the next section for complete definitions). The purpose of this article is to study, in great details, the boundedness properties of such operators in various function spaces. All what we will discuss about these operators extends to obvious and properly normalized $m$-linear generalizations involving $m$ input functions and $m+1$ families of molecules. For simplicity in the notation and ease in the flow of the article we will restrict ourselves to the bilinear case.

Second and fourth authors' research partially supported by the NSF under grant DMS 0400423.

Third author's research partially supported by the NSF under grant DMS 0503542 .

* Corresponding author: e-mail: torres@math.ku.edu, Phone: +01 (785) 864 7310, Fax: +01 (785) 8645255 
Operators of the form (1) appear as model operators and technical tools in the study of bilinear singular integrals. We revisit bilinear paraproducts motivated in part by the work of Lacey and Metcalfe in [20], which in turn is motivated by the work of Muscalu et al. in [24]. In both works, operators of the type (1) and their multiparameter versions (in the sense of non-homogeneous dilations) are studied. They are used in [24] to develop a biparameter Coifman-Meyer multiplier result, while some further end-point estimates for such operators are presented in [20]. See also [25].

As it is known, the classical Calderón-Zygmund theory does not apply to the multiparameter dilation setting in the same way it does in the one-parameter one (some weak end-point estimates are not available), and the use of paraproducts as in [24] was crucial to develop the complete set of $L^{p}$ estimates for biparameter CoifmanMeyer multipliers. Such range of estimates recovered the ones for one-parameter dilations (the only case we will treat here), where the classical Calderón-Zygmund theory approach does work and was previously developed by Kenig and Stein [19] and Grafakos and Torres [17] without the need of the paraproducts (1). An approach to one-parameter Coifman-Meyer multipliers based on paraproducts was also carried out by Gilbert and Nahmod in [13].

In this article, we retrieve the $L^{p}$ estimates for the operators of the form (1) by taking advantage of the fairly general multilinear Calderón-Zygmund theory developed in [16], [17], and [18]. See also [6], [9] and [19]. This theory also includes operators of non-convolution type as well as all their natural end-point, maximal function, and weighted estimates. Consequently, we also obtain new boundedness results on some other spaces (e.g., weighted and weak Lebesgue spaces, Hardy spaces, and Sobolev spaces) by treating the paraproducts as bilinear Calderón-Zygmund operators of non-convolution type. In addition, by using molecular decompositions, almost diagonal estimates from [11] and [16], and their discrete equivalents, we present boundedness results on products of Triebel-Lizorkin spaces.

We point out that a similar approach using molecular decomposition has been considered by Wang in [33] and [34] by fixing one of the functions in (1), say $g$, and realizing as in the classical case $T_{g}(f)=T(f, g)$ as a linear Calderón-Zygmund operator. Such an approach typically produces results of the form $T: X \times Y \rightarrow Y$, where $X$ and $Y$ are appropriate function spaces. We emphasize here the bilinear approach obtaining more general results of the form $T: X \times Y \rightarrow Z$, again for appropriately related function spaces $X, Y$, and $Z$.

Acknowledgment: This work was motivated in part by some of the mathematical interactions that took place during the visit of Bényi and Torres to the Erwin Schrödinger Institute (ESI), Vienna, Austria. They want to thank the ESI and the organizers of the special semester on Modern Methods of Time-Frequency Analysis for their support and hospitality.

\section{Notation and preliminaries}

For $\nu \in \mathbf{Z}$ and $k \in \mathbf{Z}^{n}$, let $P_{\nu k}$ be the dyadic cube

$$
P_{\nu k}=\left\{\left(x_{1}, \ldots, x_{n}\right) \in \mathbf{R}^{n}: k_{i} \leq 2^{\nu} x_{i}<k_{i}+1, i=1, \ldots, n\right\} .
$$

The lower left-corner of $P=P_{\nu k}$ is denoted by $x_{P}=x_{\nu k}=2^{-\nu} k$, its size by $|P|=2^{-\nu n}$, and its characteristic function by $\chi_{P_{\nu k}}$. The collection of all dyadic cubes is $\mathcal{D}=\left\{P_{\nu k}: \nu \in \mathbf{Z}, k \in \mathbf{Z}^{n}\right\}$.

In this article, a smooth molecule associated to $P$ is a function $\phi_{P}=\phi_{P_{\nu k}}=\phi_{\nu k}$ that satisfies

$$
\left|\partial^{\gamma} \phi_{\nu k}(x)\right| \leq \frac{C_{\gamma, N} 2^{\nu n / 2} 2^{|\gamma| \nu}}{\left(1+2^{\nu}\left|x-2^{-\nu} k\right|\right)^{N}}, \text { for all }|\gamma| \leq M \text { and some } N>n .
$$

The regularity $M$ and the decay $N>n$ will be specified in applications. For instance, if $\phi$ is in the Schwartz class $\mathcal{S}\left(\mathbf{R}^{n}\right)$ then

$$
\phi_{\nu k}(x)=2^{\nu n / 2} \phi\left(2^{\nu} x-k\right)
$$

is a molecule associated to $P_{\nu k}$ with arbitrary smoothness and decay.

A family of smooth molecules $\left\{\phi_{P}\right\}_{P \in \mathcal{D}}=\left\{\phi_{\nu k}\right\}_{\nu \in \mathbf{Z}, k \in \mathbf{Z}^{n}}$ that satisfy the additional conditions

$$
\int \phi_{\nu k}(x) x^{\gamma} d x=0, \text { for all }|\gamma| \leq L, \nu \in \mathbf{Z}, k \in \mathbf{Z}^{n},
$$


where $L$ will be specified in particular uses, will be called a family of smooth molecules with cancellation. By properly choosing $M$ and $N$ in (3) and $L$ in (5), such families become smooth molecules for the Triebel-Lizorkin space $\dot{F}_{p}^{\alpha, s}\left(\mathbf{R}^{n}\right)$ in the sense of Frazier and Jawerth [11]. Next, we recall the definition of such spaces and their (non-orthogonal) wavelet and molecular decompositions.

Let $\langle f, \phi\rangle$ be the pairing between a test function $\phi$ in $\mathcal{S}\left(\mathbf{R}^{n}\right)$ and a tempered distribution $f$ in $\mathcal{S}^{\prime}\left(\mathbf{R}^{n}\right)$. The Fourier transform of a test function is defined via $\widehat{\phi}(\xi)=\left\langle e^{-i \xi}, \phi\right\rangle$ and extended to distributions in the usual way. We will write $\psi \in B P F\left(\mathbf{R}^{n}\right)$ (BPF after "band pass filter") if $\psi$ belongs to $\mathcal{S}\left(\mathbf{R}^{n}\right), \psi$ is real-valued and radial, its Fourier transform is supported in the annulus $\left\{\xi \in \mathbf{R}^{n}: \pi / 4 \leq|\xi| \leq \pi\right\}$, and $|\widehat{\psi}(\xi)|$ is bounded away from zero on, say, $\left\{\xi \in \mathbf{R}^{n}: \pi / 4+\epsilon \leq|\xi| \leq \pi-\epsilon\right\}$ for $\epsilon>0$ small. Fix now $\psi \in B P F\left(\mathbf{R}^{n}\right)$; for $0<p, s<\infty$ and $\alpha$ real we will consider the Triebel-Lizorkin space $\dot{F}_{p}^{\alpha, s}$ with (quasi) norm given by

$$
\|f\|_{\dot{F}_{p}^{\alpha, s}\left(\mathbf{R}^{n}\right)}=\left\|\left[\sum_{\nu \in \mathbf{Z}}\left(2^{\nu \alpha}\left|f * \psi_{\nu}\right|\right)^{s}\right]^{1 / s}\right\|_{L^{p}\left(\mathbf{R}^{n}\right)},
$$

where, now, $\psi_{\nu}(x)=2^{\nu n} \psi\left(2^{\nu} x\right)$. Of main interest to us are the particular cases of

(i) the Lebesgue spaces, $\|f\|_{\dot{F}_{p}^{0,2}\left(\mathbf{R}^{n}\right)} \approx\|f\|_{L^{p}\left(\mathbf{R}^{n}\right)}$ for $1<p<\infty$;

(ii) the Hardy spaces, $\|f\|_{\dot{F}_{p}^{0,2}\left(\mathbf{R}^{n}\right)} \approx\|f\|_{H^{p}\left(\mathbf{R}^{n}\right)}$ for $0<p \leq 1$ and;

(iii) the homogeneous Sobolev spaces, $\|f\|_{\dot{F}_{p}^{\alpha, 2}\left(\mathbf{R}^{n}\right)} \approx\|f\|_{\dot{L}^{\alpha, p}\left(\mathbf{R}^{n}\right)}$ for $1<p<\infty$, where $\|f\|_{\dot{L}_{\alpha}^{p}\left(\mathbf{R}^{n}\right)}=$ $\left\||\nabla|^{\alpha} f\right\|_{L^{p}\left(\mathbf{R}^{n}\right)}$ with $\widehat{\left.\nabla \nabla\right|^{\alpha} f}=|\xi|^{\alpha} \widehat{f}$ defined a priori for all $\alpha$ if $f$ is in

$$
\mathcal{S}_{0}\left(\mathbf{R}^{n}\right)=\left\{f \in \mathcal{S}\left(\mathbf{R}^{n}\right): \partial^{\gamma} \widehat{f}(0)=0, \text { for all } \gamma \in \mathbf{N}_{0}^{n}\right\}
$$

We also note that the familiar in-homogeneous Sobolev spaces $L_{\alpha}^{p}$, with norm given by $\|f\|_{L_{\alpha}^{p}\left(\mathbf{R}^{n}\right)}=\|(I-$ $\Delta)^{\alpha / 2} f \|_{L^{p}\left(\mathbf{R}^{n}\right)}$ and where $\left(I-\widehat{-\Delta)^{\alpha} / 2} f=\left(1+|\xi|^{2}\right)^{\alpha / 2} \widehat{f}\right.$, satisfy $L_{\alpha}^{p}=L^{p} \cap \dot{L}_{\alpha}^{p}$ for $1<p<\infty$ and $\alpha>0$. The Littlewood-Paley characterization (6) of the Lebesgue, Hardy, and Sobolev spaces is nowadays well-known. For more on the general scale of spaces $\dot{F}_{p}^{\alpha, s}\left(\mathbf{R}^{n}\right)$ see for example [32]. Following [11], if one also requires $\psi$ to satisfy

$$
\sum_{\nu \in \mathbf{Z}}\left|\widehat{\psi}\left(2^{\nu} \xi\right)\right|^{2}=1, \quad \xi \neq 0,
$$

then one can write the wavelet-type expansion

$$
f=\sum_{\nu \in \mathbf{Z}} \sum_{k \in \mathbf{Z}^{n}}\left\langle f, \psi_{\nu k}\right\rangle \psi_{\nu k}
$$

where $\psi_{\nu k}(x)=2^{\nu n / 2} \psi\left(2^{\nu} x-k\right)$, to obtain

$$
\|f\|_{\dot{F}_{p}^{\alpha, s}\left(\mathbf{R}^{n}\right)} \approx\left\|\left\{\left\langle f, \psi_{\nu k}\right\rangle\right\}\right\|_{\dot{f}_{p}^{\alpha, s}},
$$

where $\dot{f}_{p}^{\alpha, s}$ denotes the space of sequences $\left\{u_{\nu k}\right\}_{\nu \in \mathbf{Z}, k \in \mathbf{Z}^{n}}$ for which

$$
\left\|\left\{u_{\nu k}\right\}_{\nu \in \mathbf{Z}, k \in \mathbf{Z}^{n}}\right\|_{f_{p}^{\alpha, s}}=\left\|\left[\sum_{\nu \in \mathbf{Z}} \sum_{k \in \mathbf{Z}^{n}}\left(\left|u_{\nu k}\right| 2^{\nu(\alpha+n / 2)} \chi_{P_{\nu k}}\right)^{s}\right]^{1 / s}\right\|_{L^{p}\left(\mathbf{R}^{n}\right)}<\infty .
$$

Note that these are not orthogonal wavelets as in [23], but they still characterize the function spaces used. Moreover, if $\left\{\phi_{Q}\right\}_{Q \in \mathcal{D}}$ is a general family of molecules for $\dot{F}_{p}^{\alpha, s}\left(\mathbf{R}^{n}\right)$ with enough decay, regularity, and cancellation (see Remark 2.1 below), and if $\left\{c_{Q}\right\}_{Q \in \mathcal{D}}=\left\{c_{\nu k}\right\}_{\nu \in \mathbf{Z}, k \in \mathbf{Z}^{n}}$ is a sequence of numbers in $\dot{f}_{p}^{\alpha, s}$, then

$$
f=\sum_{Q \in \mathcal{D}} c_{Q} \phi_{Q}
$$


is in $\dot{F}_{p}^{\alpha, s}$ and satisfies

$$
\|f\|_{\dot{F}_{p}^{\alpha, s}\left(\mathbf{R}^{n}\right)} \leq C\left\|\left\{c_{P}\right\}\right\|_{\dot{f}_{p}^{\alpha, s}},
$$

with $C$ depending only on the family of molecules.

Remark 2.1 In general, the parameters $N, M, L$ in the definition of the molecules for a particular space $\dot{F}_{p}^{\alpha, s}\left(\mathbf{R}^{n}\right)$ depend on $\alpha, p, s$ and the dimension $n$. We will not use the sharpest possible values but we note that $N>n / \min (1, p, s), M=\lfloor\alpha\rfloor+1$, and $L=\max \left(\left\lfloor\frac{n}{\min (1, p, s)}-n-\alpha\right\rfloor,-1\right)$, with the convention that (5) is void if $L<0$, suffice if $\alpha \geq 0$. For $\alpha<0$ one also needs to add (3) with $N>n / \min (1, p, s)-\alpha$ when $|\gamma|=0$. In particular, one can use:

(i) $N>n, M=1$, and $L=0$ for the Lebesgue spaces $L^{p}\left(\mathbf{R}^{n}\right)$ when $1<p<\infty$;

(ii) $N>n / p, M=1$, and the typical cancellation $L=\lfloor n(1 / p-1)\rfloor$ for the Hardy spaces $H^{p}\left(\mathbf{R}^{n}\right)$ when $0<p \leq 1$ and

(iii) $N>n, M=\lfloor\alpha\rfloor+1$, and no cancellation for the Sobolev spaces $\dot{L}_{\alpha}^{p}\left(\mathbf{R}^{n}\right)$ when $1<p<\infty$ and $\alpha>0$.

Remark 2.2 There are dual counterparts to the above statement and the estimate (12) involving what we will call dual molecules. A family of functions $\left\{\phi_{P}\right\}_{P \in \mathcal{D}}=\left\{\phi_{\nu k}\right\}_{\nu \in \mathbf{Z}, k \in \mathbf{Z}^{n}}$ is a family of dual molecules for $\dot{F}_{p}^{\alpha, s}\left(\mathbf{R}^{n}\right)$ if the functions $\phi_{P}$ satisfy the conditions (3) and (5) with $N>n / \min (1, p, s)$ and $M=$ $\max \left(\left\lfloor\frac{n}{\min (1, p, s)}-n-\alpha\right\rfloor+1,0\right)$ and $L=\lfloor\alpha\rfloor$, and the additional decay condition

$$
\left|\phi_{\nu k}(x)\right| \leq \frac{C 2^{\nu n / 2}}{\left(1+2^{\nu}\left|x-2^{-\nu} k\right|\right)^{\max (N, N+n+\alpha-n / \min (1, p, s))}} .
$$

This last condition is included in (3) if $p, s \geq 1$ and $\alpha=0$. If $\left\{\phi_{P}\right\}$ is a family of dual molecules for $\dot{F}_{p}^{\alpha, s}\left(\mathbf{R}^{n}\right)$, then

$$
\left\|\left\{\left\langle f, \phi_{P}\right\rangle\right\}\right\|_{\dot{f}_{p}^{\alpha, s}} \leq C\|f\|_{\dot{F}_{p}^{\alpha, s}\left(\mathbf{R}^{n}\right)} .
$$

See [11] and [12] for more about the molecular decompositions described.

The wavelet characterization of the Triebel-Lizorkin spaces permits the study of linear and bilinear operators by examining their associated discrete matrices or tensors on the spaces of coefficients. The approach is wellknown. In the linear case we can write

$$
T(f)=\sum_{P \in \mathcal{D}}\left\langle f, \psi_{P}\right\rangle T\left(\psi_{P}\right)=\sum_{Q \in \mathcal{D}} \sum_{P \in \mathcal{D}}\left\langle f, \psi_{P}\right\rangle\left\langle T\left(\psi_{P}\right), \psi_{Q}\right\rangle \psi_{Q}
$$

and reduce the study of $T$ on $\dot{F}_{p}^{\alpha, s}\left(\mathbf{R}^{n}\right)$ to that of the matrix $A=\left\{a_{Q P}\right\}_{Q, P \in \mathcal{D}}=\left\{\left\langle T\left(\psi_{P}\right), \psi_{Q}\right\rangle\right\}_{Q, P \in \mathcal{D}}$ on $\dot{f}_{p}^{\alpha, s}$. Moreover, since the spaces $\dot{f}_{p}^{\alpha, s}$ are defined in terms of the size of the sequences, it is enough to obtain boundedness results for the matrix $\left\{\left|a_{Q P}\right|\right\}_{Q, P \in \mathcal{D}}$. There exist sufficient conditions in terms of the size of the entries of this matrix which ensure its boundedness on $\dot{f}_{p}^{\alpha, s}$ and they are known as almost diagonal estimates [11]. These are decay estimates in $\left|a_{Q P}\right|$ for $P$ and $Q$ far from each other in an appropriate sense. We recall the following fact

Theorem 2.3 (Frazier-Jawerth, Lemmas 3.6 and 3.8 in [11]) Let $\left\{\psi_{Q}\right\}$ be a family of molecules obtained by dilations and translations of a fixed function $\psi \in B P F\left(\mathbf{R}^{n}\right)$, that is, $\psi_{Q}(x)=\psi_{\nu k}(x)=2^{\nu n / 2} \psi\left(2^{\nu} x-k\right)$, where $Q=Q_{\nu k}$.

(i) If $\left\{\phi_{P}\right\}$ is a family of smooth molecules for the space $\dot{F}_{p}^{\alpha, s}\left(\mathbf{R}^{n}\right)$, then $A=\left\{a_{P Q}\right\}=\left\{\left\langle\phi_{P}, \psi_{Q}\right\rangle\right\}$ is a bounded operator on $\dot{f}_{p}^{\alpha, s}$.

(ii) If $\left\{\phi_{P}\right\}$ is a family of dual molecules for the space $\dot{F}_{p}^{\alpha, s}\left(\mathbf{R}^{n}\right)$, then $A=\left\{a_{P Q}\right\}=\left\{\left\langle\phi_{P}, \psi_{Q}\right\rangle\right\}$ is a bounded operator on $\dot{f}_{p}^{\alpha, s}$. 
This discretization technique extends to the bilinear setting. For instance, if we consider $\psi \in B P F\left(\mathbf{R}^{n}\right)$ and again $\psi_{\nu k}(x)=2^{\nu n / 2} \psi\left(2^{\nu} x-k\right)$, we can now represent a bilinear operator in the form

$$
T(f, g)=\sum_{\lambda, m} \sum_{\mu, l} \sum_{\nu, k}\left\langle T\left(\psi_{\nu k}, \psi_{\mu l}\right), \psi_{\lambda m}\right\rangle\left\langle f, \psi_{\nu k}\right\rangle\left\langle g, \psi_{\mu l}\right\rangle \psi_{\lambda m}
$$

and reduce the study of $T$ on products of $\dot{F}_{p}^{\alpha, s}$ spaces to that of

$$
A=\{a(\lambda m, \nu k, \mu l)\}=\left\{\left\langle T\left(\psi_{\nu k}, \psi_{\mu l}\right), \psi_{\lambda m}\right\rangle\right\}
$$

on products of $\dot{f}_{p}^{\alpha, s}$ spaces. Bilinear almost diagonal estimates are available and depend on the spaces used (see Theorem 5.4 below). The almost diagonal estimates can be extended to the general multilinear setting as in the work of Bényi and Tzirakis [4]. Finally, we need to recall one more background result concerning bilinear Calderón-Zygmund operators. Let $T$ be a bilinear operator, say initially defined on pairs of test functions into the space of distributions, whose distributional kernel coincides away from the diagonal $\Omega=\left\{(x, y, z) \in\left(\mathbf{R}^{n}\right)^{3}\right.$ : $x=y=z\}$ with a function $K(x, y, z)$ locally integrable in $\left(\mathbf{R}^{n}\right)^{3} \backslash \Omega$ that satisfies in that set the size condition

$$
|K(x, y, z)| \leq C(|x-y|+|x-z|+|y-z|)^{-2 n},
$$

and the regularity condition

$$
\left|K(x, y, z)-K\left(x^{\prime}, y, z\right)\right| \leq C \frac{\left|x-x^{\prime}\right|^{\varepsilon}}{(|x-y|+|x-z|+|y-z|)^{2 n+\varepsilon}},
$$

for some $\varepsilon \in(0,1]$ whenever $\left|x-x^{\prime}\right| \leq \frac{1}{2} \max \{|x-y|,|x-z|\}$. For symmetry and interpolation purposes we also require that the functions $K_{1}(x, y, z)=K(y, x, z)$ and $K_{2}(x, y, z)=K(z, y, x)$ also satisfy (16). We say that such a kernel $K(x, y, z)$ is a bilinear Calderón-Zygmund kernel and that such an operator $T$ is a bilinear Calderón-Zygmund operator if it extends to a bounded operator from $L^{p_{0}} \times L^{q_{0}}$ into $L^{r_{0}}$ for some $1<p_{0}, q_{0}<\infty$ and $1 / p_{0}+1 / q_{0}=1 / r_{0} \leq 1$.

\section{Continuous and discrete almost orthogonality estimates}

Atomic and molecular decompositions have been extensively used in the literature to study operators of CalderónZygmund type on the scale of Triebel-Lizorkin spaces. See [29] in the linear case and [1] in the bilinear situation for more results, complete accounts, and further references. A key ingredient in the approach is that molecules associated to different cubes are almost orthogonal. In the linear case, this phenomenon is quantified in the following propositions.

Proposition 3.1 (Frazier-Jawerth, Appendix B in [11]) Suppose that $\varphi_{\nu}$ and $\varphi_{\mu}$ are functions defined on $\mathbf{R}^{n}$ satisfying for some $x_{\nu}, x_{\mu}$ in $\mathbf{R}^{n}$

$$
\begin{aligned}
& \left|\varphi_{\nu}(x)\right| \leq \frac{2^{\nu n / 2}}{\left(1+2^{\nu}\left|x-x_{\nu}\right|\right)^{N}}, \\
& \left|\varphi_{\mu}(x)\right| \leq \frac{2^{\mu n / 2}}{\left(1+2^{\mu}\left|x-x_{\mu}\right|\right)^{N}},
\end{aligned}
$$

for some constant $N>n$. Then, there exists a constant $C=C(n, N)>0$ such that the following estimate is valid

$$
\int_{\mathbf{R}^{n}}\left|\varphi_{\nu}(x)\right|\left|\varphi_{\mu}(x)\right| d x \leq C \frac{2^{-|\nu-\mu| n / 2}}{\left(1+2^{\min (\mu, \nu)}\left|x_{\nu}-x_{\mu}\right|\right)^{N}} .
$$

Proposition 3.2 (Frazier-Jawerth, Appendix B in [11]) Suppose that $\varphi_{\nu}$ and $\varphi_{\mu}$ are functions defined on $\mathbf{R}^{n}$ such that for some $x_{\nu}, x_{\mu}$ in $\mathbf{R}^{n}$, some $N_{1}>n+L+1$ with L a non-negative integer, and some $N_{2}>n$ the following conditions hold:

$$
\left|\varphi_{\nu}(x)\right| \leq \frac{2^{\nu n / 2}}{\left(1+2^{\nu}\left|x-x_{\nu}\right|\right)^{\max \left(N_{1}, N_{2}\right)}}
$$




$$
\int_{\mathbf{R}^{n}} \varphi_{\nu}(x) x^{\gamma} d x=0 \quad \text { for all }|\gamma| \leq L
$$

and

$$
\left|\partial_{x}^{\gamma} \varphi_{\mu}(x)\right| \leq \frac{2^{\mu|\gamma|} 2^{\mu n / 2}}{\left(1+2^{\mu}\left|x-x_{\mu}\right|\right)^{N_{2}}} \quad \text { for all }|\gamma| \leq L+1 .
$$

Then, for $\nu \geq \mu$ there exists a constant $C=C\left(N_{1}, N_{2}, L\right)>0$ such that the following estimate is valid

$$
\left|\int_{\mathbf{R}^{n}} \varphi_{\nu}(x) \varphi_{\mu}(x) d x\right| \leq C \frac{2^{-(\nu-\mu)(L+1+n / 2)}}{\left(1+2^{\mu}\left|x_{\nu}-x_{\mu}\right|\right)^{N_{2}}} .
$$

For three real numbers, $a_{1}, a_{2}, a_{3}$, we denote by $\operatorname{med}\left(a_{1}, a_{2}, a_{3}\right)$ one of the $a_{j}$ 's that satisfies $\min \left(a_{1}, a_{2}, a_{3}\right) \leq$ $a_{j} \leq \max \left(a_{1}, a_{2}, a_{3}\right)$. In the bilinear case we have

Proposition 3.3 (Grafakos-Torres, Proposition 3 in [16]) Suppose that $\varphi_{\nu}, \varphi_{\mu}, \varphi_{\lambda}$ are functions defined on $\mathbf{R}^{n}$ satisfying the following estimates for all $x \in \mathbf{R}^{n}$

$$
\begin{aligned}
& \left|\varphi_{\nu}(x)\right| \leq \frac{2^{\nu n / 2}}{\left(1+2^{\nu}\left|x-x_{\nu}\right|\right)^{5 N}}, \\
& \left|\varphi_{\mu}(x)\right| \leq \frac{2^{\mu n / 2}}{\left(1+2^{\mu}\left|x-x_{\mu}\right|\right)^{5 N}}, \\
& \left|\varphi_{\lambda}(x)\right| \leq \frac{2^{\lambda n / 2}}{\left(1+2^{\lambda}\left|x-x_{\lambda}\right|\right)^{5 N}},
\end{aligned}
$$

for some $x_{\nu}, x_{\mu}, x_{\lambda}$ in $\mathbf{R}^{n}$ and some $N>n$. Then the following estimate is valid

$$
\begin{aligned}
& \int_{\mathbf{R}^{n}}\left|\varphi_{\nu}(x)\right|\left|\varphi_{\mu}(x)\right|\left|\varphi_{\lambda}(x)\right| d x \leq \\
& \frac{C 2^{-\max (\mu, \nu, \lambda) n / 2} 2^{\operatorname{med}(\mu, \nu, \lambda) n / 2} 2^{\min (\mu, \nu, \lambda) n / 2}}{\left(\left(1+2^{\min (\nu, \mu)}\left|x_{\nu}-x_{\mu}\right|\right)\left(1+2^{\min (\mu, \lambda)}\left|x_{\mu}-x_{\lambda}\right|\right)\left(1+2^{\min (\lambda, \nu)}\left|x_{\lambda}-x_{\nu}\right|\right)\right)^{N}}
\end{aligned}
$$

for some $C=C(N, n)>0$.

The rest of this section is devoted to proving a discrete version of Proposition 3.3 (Proposition 3.6 below) that will suit the discrete nature of the paraproduct (1). The proof is similar to the one in the continuous case and we include it for completeness since it will be repeatedly utilized. We start with two lemmas.

Lemma 3.4 Let $\beta \geq 0, N>n+\beta n+1$ and $a, b, c \geq 0$ with $a+b+c>0$. Then,

$$
\sum_{\nu \in \mathbf{Z}} \frac{2^{n \nu \beta} 2^{n \nu}}{\left(1+2^{\nu} a\right)^{N}\left(1+2^{\nu} b\right)^{N}\left(1+2^{\nu} c\right)^{N}} \leq C \frac{1}{(a+b+c)^{n+n \beta}},
$$

where $C$ depends only on $n, \beta$, and $N$.

Proof. Given $d>0, \beta \geq 0$ and $N>n+n \beta+1$, it easily follows that

$$
\sum_{\nu \in \mathbf{Z}} \frac{2^{n \nu \beta} 2^{n \nu}}{\left(1+2^{\nu} d\right)^{N}} \leq C \frac{1}{d^{n+n \beta}}
$$

where $C$ depends only on $\beta, n$ and $N$. Next, just notice that

$$
\begin{aligned}
& \left(1+2^{\nu} a\right)^{N}\left(1+2^{\nu} b\right)^{N}\left(1+2^{\nu} c\right)^{N} \\
& =\left(1+(a+b+c) 2^{\nu}+(a b+b c+c a) 2^{2 \nu}+a b c 2^{3 \nu}\right)^{N} \\
& \geq\left(1+(a+b+c) 2^{\nu}\right)^{N}
\end{aligned}
$$

and use (27) with $d=a+b+c$. 
Lemma 3.5 For every $N>n+1$ there exists a constant $C$, depending only on $n$ and $N$, such that for any $\gamma \in \mathbf{Z}, a, R>0$, and $x, y \in \mathbf{R}^{n}$ the following inequality holds

$$
\sum_{\left\{k \in \mathbf{Z}^{n}:\left|2^{-\gamma} k-x\right| \leq R\right\}} \frac{1}{\left(1+a\left|2^{-\gamma} k-y\right|\right)^{N}} \leq \frac{C 2^{\gamma n}}{(1+a|x-y|)^{N}} \max \left\{\frac{(a R)^{N}}{a^{n}}, R^{n}\right\} .
$$

Proof. Set $S=\left\{k \in \mathbf{Z}^{n}:\left|2^{-\gamma} k-x\right| \leq R\right\}$ and notice that the cardinality of $S$ is at most $c_{n}\left(2^{\gamma} R\right)^{n}$ for a dimensional constant $c_{n}$. In order to prove the lemma, consider first the case $a R \leq 10$. If $a|x-y| \leq 20$, then

$$
1 \leq \frac{21^{N}}{(1+a|x-y|)^{N}}
$$

and

$$
\sum_{\left\{k \in \mathbf{Z}^{n}:\left|2^{-\gamma} k-x\right| \leq R\right\}} \frac{1}{\left(1+a\left|2^{-\gamma} k-y\right|\right)^{N}} \leq \# S \leq c_{n}\left(2^{\gamma} R\right)^{n} \leq \frac{c_{n} 21^{N} 2^{\gamma n}}{(1+a|x-y|)^{N}} R^{n} .
$$

If $a|x-y| \geq 20$ then for $k \in S$ we have

$$
\begin{aligned}
|x-y| & \leq\left|2^{-\gamma} k-x\right|+\left|2^{-\gamma} k-y\right| \leq R+\left|2^{-\gamma} k-y\right| \\
& \leq \frac{10}{a}+\left|2^{-\gamma} k-y\right| \leq \frac{1}{2}|x-y|+\left|2^{-\gamma} k-y\right| .
\end{aligned}
$$

Therefore,

$$
\sum_{\left\{k \in \mathbf{Z}^{n}:\left|2^{-\gamma} k-x\right| \leq R\right\}} \frac{1}{\left(1+a\left|2^{-\gamma} k-y\right|\right)^{N}} \leq \frac{2^{N} \# S}{(1+a|x-y|)^{N}} \leq \frac{c_{n} 2^{N} 2^{\gamma n}}{(1+a|x-y|)^{N}} R^{n} .
$$

In the case $a R>10$ we define

$$
A_{1}=\left\{k \in \mathbf{Z}^{n}:\left|2^{-\gamma} k-y\right| \geq|x-y|\right\} \cap S,
$$

and $A_{2}=S \backslash A_{1}$. Summing over $A_{1}$ gives

$$
\sum_{k \in A_{1}} \frac{1}{\left(1+a\left|2^{-\gamma} k-y\right|\right)^{N}} \leq \frac{\# A_{1}}{(1+a|x-y|)^{N}} \leq \frac{c_{n} 2^{\gamma n}}{(1+a|x-y|)^{N}} R^{n} .
$$

Before summing over $A_{2}$ notice that

$$
\begin{aligned}
& \sum_{k \in \mathbf{Z}^{n}} \frac{1}{\left(1+a\left|2^{-\gamma} k-y\right|\right)^{N}}=\sum_{j \in \mathbf{Z}} \sum_{\left\{k \in \mathbf{Z}^{n}: 2^{j} \leq\left|2^{-\gamma} k-y\right|<2^{j+1}\right\}} \frac{1}{\left(1+a\left|2^{-\gamma} k-y\right|\right)^{N}} \\
& \leq \sum_{j \in \mathbf{Z}} \frac{\#\left\{k \in \mathbf{Z}^{n}: 2^{j} \leq\left|2^{-\gamma} k-y\right|<2^{j+1}\right\}}{\left(1+a 2^{j}\right)^{N}} \leq c_{n} 2^{n} 2^{\gamma n} \sum_{j \in \mathbf{Z}} \frac{2^{j n}}{\left(1+a 2^{j}\right)^{N}} \\
& \leq C_{1}(n, N) \frac{2^{\gamma n}}{a^{n}},
\end{aligned}
$$

where the last inequality follows from (27) with $\beta=0$ and $d=a$. To sum over $A_{2}$ we first consider the case $|x-y| \leq 2 R$, so that

$$
1+a|x-y| \leq 1+2 a R \leq 3 a R
$$

and consequently

$$
\begin{aligned}
\sum_{k \in A_{2}} \frac{1}{\left(1+a\left|2^{-\gamma} k-y\right|\right)^{N}} & \leq \sum_{k \in \mathbf{Z}^{n}} \frac{1}{\left(1+a\left|2^{-\gamma} k-y\right|\right)^{N}} \leq C_{1}(n, N) \frac{2^{\gamma n}}{a^{n}} \\
& \leq \frac{C_{1}(n, N) 3^{N} 2^{\gamma n}}{(1+a|x-y|)^{N}} \frac{(a R)^{N}}{a^{n}} .
\end{aligned}
$$


If $|x-y|>2 R$, we obtain again

$$
|x-y| \leq\left|x-2^{-\gamma} k\right|+\left|2^{-\gamma} k-y\right| \leq R+\left|2^{-\gamma} k-y\right| \leq \frac{1}{2}|x-y|+\left|2^{-\gamma} k-y\right| .
$$

Hence,

$$
\begin{aligned}
\sum_{k \in A_{2}} \frac{1}{\left(1+a\left|2^{-\gamma} k-y\right|\right)^{N}} & \leq 2^{N} \sum_{k \in A_{2}} \frac{1}{(1+a|x-y|)^{N}} \leq \frac{2^{N} \# A_{2}}{(1+a|x-y|)^{N}} \\
& \leq \frac{c_{n} 2^{N} 2^{\gamma n}}{(1+a|x-y|)^{N}} R^{n} .
\end{aligned}
$$

The lemma then follows with $C=\max \left\{C_{1}(n, N) 3^{N}, c_{n} 21^{N}\right\}$.

Proposition 3.6 For every $N>n+1$ there is a constant $C$, depending only on $N$ and $n$, such that for any $\gamma, \nu, \mu, \lambda \in \mathbf{Z}$ and any $x, y, z \in \mathbf{R}^{n}$ the following inequality holds

$$
\begin{aligned}
& \sum_{k \in \mathbf{Z}^{n}} \frac{2^{-\gamma n} 2^{\nu n / 2} 2^{\mu n / 2} 2^{\lambda n / 2}}{\left[\left(1+2^{\nu}\left|x-2^{-\gamma} k\right|\right)\left(1+2^{\mu}\left|y-2^{-\gamma} k\right|\right)\left(1+2^{\lambda}\left|z-2^{-\gamma} k\right|\right)\right]^{5 N}} \\
& \leq \frac{C 2^{-\max (\mu, \nu, \lambda) n / 2} 2^{\operatorname{med}(\mu, \nu, \lambda) n / 2} 2^{\min (\mu, \nu, \lambda) n / 2}}{\left(\left(1+2^{\min (\nu, \mu)}|x-y|\right)\left(1+2^{\min (\mu, \lambda)}|y-z|\right)\left(1+2^{\min (\nu, \lambda)}|x-z|\right)\right)^{N}} .
\end{aligned}
$$

Proof. By symmetry and without loss of generality we can assume that $\mu \leq \lambda \leq \nu$. We split the sum as follows

$$
\begin{aligned}
& \sum_{k \in \mathbf{Z}^{n}} \frac{2^{-\gamma n} 2^{\nu n / 2} 2^{\mu n / 2} 2^{\lambda n / 2}}{\left[\left(1+2^{\nu}\left|x-2^{-\gamma} k\right|\right)\left(1+2^{\mu}\left|y-2^{-\gamma} k\right|\right)\left(1+2^{\lambda}\left|z-2^{-\gamma} k\right|\right)\right]^{5 N}} \\
& =\sum_{\substack{s, t=0 \\
\mid}}^{\infty} \sum_{\substack{\left|2^{-\gamma} k-x\right| \sim 2^{-\nu} 2^{t} \\
\left|2^{-\gamma}-z\right| \sim 2^{-\lambda} 2^{s}}} \frac{2^{-\gamma n} 2^{\nu n / 2} 2^{\mu n / 2} 2^{\lambda n / 2}}{\left[\left(1+2^{\nu}\left|x-2^{-\gamma} k\right|\right)\left(1+2^{\mu}\left|y-2^{-\gamma} k\right|\right)\left(1+2^{\lambda}\left|z-2^{-\gamma} k\right|\right)\right]^{5 N}}
\end{aligned}
$$

where the notation $\left|2^{-\gamma} k-x\right| \sim 2^{-\nu} 2^{t}$ means $2^{-\nu} 2^{t} \leq\left|2^{-\gamma} k-x\right|<2^{-\nu} 2^{t+1}$ for $t \geq 1$ and $\left|2^{-\gamma} k-x\right|<2^{-\nu} 2^{t}$ if $t=0$. Similarly with $\left|2^{-\gamma} k-z\right| \sim 2^{-\lambda} 2^{s}$. The conditions $\left|2^{-\gamma} k-x\right| \sim 2^{-\nu} 2^{t}$ and $\left|2^{-\gamma} k-z\right| \sim 2^{-\lambda} 2^{s}$ clearly imply

$$
\frac{1}{\left(1+2^{\nu}\left|2^{-\gamma} k-x\right|\right)^{5 N}} \leq \frac{1}{2^{5 t N}}, \quad t=0,1,2, \ldots
$$

and

$$
\frac{1}{\left(1+2^{\lambda}\left|2^{-\gamma} k-z\right|\right)^{5 N}} \leq \frac{1}{2^{5 s N}}, \quad s=0,1,2, \ldots
$$

They also imply, together with $\mu \leq \lambda \leq \nu$,

$$
1+2^{\lambda}|x-z| \leq 5 \max \left(2^{t}, 2^{s}\right)
$$

and

$$
1+2^{\mu}|y-z| \leq 5 \max \left(2^{t}, 2^{s}\right)\left(1+2^{\mu}|x-y|\right)
$$

Indeed,

$$
\begin{aligned}
1+2^{\lambda}|x-z| & \leq 1+2^{\lambda}\left(\left|x-2^{-\gamma} k\right|+\left|2^{-\gamma} k-z\right|\right) \leq 1+2^{\lambda}\left(2^{-\nu} 2^{t+1}+2^{-\lambda} 2^{s+1}\right) \\
& =1+2^{\lambda-\nu} 2^{t+1}+2^{s+1} \leq 1+2\left(2^{t}+2^{s}\right)
\end{aligned}
$$


and (31) follows. On the other hand,

$$
\begin{aligned}
1+2^{\mu}|z-y| & \leq 1+2^{\mu}|x-z|+2^{\mu}|y-x| \\
& \leq 1+2^{\mu}\left|x-2^{-\gamma} k\right|+2^{\mu}\left|2^{-\gamma} k-z\right|+2^{\mu}|y-x| \\
& \leq 1+2^{\mu}\left(2^{-\nu} 2^{t+1}+2^{-\lambda} 2^{s+1}\right)+2^{\mu}|y-x| \\
& \leq 1+4 \max \left(2^{t}, 2^{s}\right)+2^{\mu}|y-x| \\
& \leq 5\left(1+2^{\mu}|y-x|\right) \max \left(2^{t}, 2^{s}\right)
\end{aligned}
$$

and (32) follows. Using (29) and (30) we obtain

$$
\begin{aligned}
& \sum_{s, t=0}^{\infty} \sum_{\substack{\left|2^{-\gamma} k-x\right| \sim 2^{-\nu} 2^{t} \\
\left|2^{-\gamma} k-z\right| \sim 2^{-\lambda} 2^{s}}} \frac{2^{-\gamma n} 2^{\nu n / 2} 2^{\mu n / 2} 2^{\lambda n / 2}}{\left[\left(1+2^{\nu}\left|x-2^{-\gamma} k\right|\right)\left(1+2^{\mu}\left|y-2^{-\gamma} k\right|\right)\left(1+2^{\lambda}\left|z-2^{-\gamma} k\right|\right)\right]^{5 N}} \\
& \leq 2^{-\gamma n} 2^{(\nu+\lambda) n / 2} \sum_{s, t=0}^{\infty} 2^{-5 N(s+t)} \sum_{k \in \mathbf{Z}^{n}:\left|2^{-\gamma} k-x\right| \leq 2^{-\nu} 2^{t}} \frac{2^{\mu n / 2}}{\left(1+2^{\mu}\left|y-2^{-\gamma} k\right|\right)^{5 N}} \\
& \leq 2^{-\gamma n} 2^{(\nu+\lambda) n / 2} \sum_{s, t=0}^{\infty} 2^{-5 N(s+t)} \sum_{k \in \mathbf{Z}^{n}:\left|2^{-\gamma} k-x\right| \leq 2^{-\nu} 2^{t}} \frac{2^{\mu n / 2}}{\left(1+2^{\mu}\left|y-2^{-\gamma} k\right|\right)^{2 N}} \\
& \leq C 2^{-\gamma n} 2^{(\nu+\lambda) n / 2} \sum_{s, t=0}^{\infty} \frac{2^{-5 N(s+t)} 2^{\gamma n} 2^{\mu n / 2}}{\left(1+2^{\mu}|x-y|\right)^{2 N}} \max \left(\frac{2^{2 N(\mu-\nu+t)}}{2^{\mu n}}, 2^{n(t-\nu)}\right) \quad \text { by Lemma } 3.5 \\
& \leq C 2^{(-\nu+\mu+\lambda) n / 2} \sum_{s, t=0}^{\infty} \frac{2^{-5 N(s+t)} 2^{2 N t}}{\left(1+2^{\mu}|x-y|\right)^{N}\left(1+2^{\mu}|x-y|\right)^{N}} \quad \text { since } N>n \text { and } \nu \geq \mu \\
& \leq C 2^{(-\nu+\mu+\lambda) n / 2} \sum_{s, t=0}^{\infty} \frac{2^{-5 N(s+t)} 2^{2 N t} \max \left(2^{t}, 2^{s}\right)^{N}}{\left(1+2^{\mu}|x-y|\right)^{N}\left(1+2^{\mu}|y-z|\right)^{N}} \quad \text { by }(32) \\
& \leq C 2^{(-\nu+\mu+\lambda) n / 2} \sum_{s, t=0}^{\infty} \frac{2^{-5 N(s+t)} 2^{2 N t} \max \left(2^{t}, 2^{s}\right)^{N} \max \left(2^{t}, 2^{s}\right)^{N}}{\left(1+2^{\mu}|x-y|\right)^{N}\left(1+2^{\mu}|y-z|\right)^{N}\left(1+2^{\lambda}|x-z|\right)^{N}} \quad \text { by }(31) \\
& =\frac{C 2^{(-\nu+\mu+\lambda) n / 2}}{\left(1+2^{\mu}|x-y|\right)^{N}\left(1+2^{\mu}|y-z|\right)^{N}\left(1+2^{\lambda}|x-z|\right)^{N}},
\end{aligned}
$$

since the sum converges to a constant depending only on $N$.

\section{Boundedness results for bilinear paraproducts}

Theorem 4.1 (Paraproducts as bilinear Calderón-Zygmund operators) Let $\left\{\phi_{Q}^{1}\right\},\left\{\phi_{Q}^{2}\right\},\left\{\phi_{Q}^{3}\right\}$ be three families of molecules satisfying (3) with $N>10 n+10$ and $M=1$. Also, suppose that at least two of the families satisfy (5) with $L=0$. Then the paraproduct

$$
T(f, g)=\sum_{Q \in \mathcal{D}}|Q|^{-1 / 2}\left\langle f, \phi_{Q}^{1}\right\rangle\left\langle g, \phi_{Q}^{2}\right\rangle \phi_{Q}^{3}, \quad f, g \in \mathcal{S}\left(\mathbf{R}^{n}\right),
$$

has a bilinear Calderón-Zygmund kernel and

$$
\|T(f, g)\|_{L^{1}\left(\mathbf{R}^{n}\right)} \leq C\|f\|_{L^{2}\left(\mathbf{R}^{n}\right)}\|g\|_{L^{2}\left(\mathbf{R}^{n}\right)} .
$$

In particular, $T$ is a bilinear Calderón-Zygmund operator. 
Using Theorem 4.1 we recover the following known estimates. See e.g. [13], [14], and [28].

Theorem 4.2 (Paraproducts on Lebesgue spaces) Let $\left\{\phi_{Q}^{1}\right\},\left\{\phi_{Q}^{2}\right\},\left\{\phi_{Q}^{3}\right\}$ be three families of molecules satisfying (3) with $N>10 n+10$ and $M=1$. Also, suppose that at least two of the families satisfy (5) with $L=0$. Then the paraproduct

$$
T(f, g)=\sum_{Q \in \mathcal{D}}|Q|^{-1 / 2}\left\langle f, \phi_{Q}^{1}\right\rangle\left\langle g, \phi_{Q}^{2}\right\rangle \phi_{Q}^{3}, \quad f, g \in \mathcal{S}\left(\mathbf{R}^{n}\right),
$$

satisfies the following boundedness properties.

(i) if $1 / p+1 / q=1 / r$ and $1<p, q<\infty$, then

$$
\|T(f, g)\|_{L^{r}\left(\mathbf{R}^{n}\right)} \leq C\|f\|_{L^{p}\left(\mathbf{R}^{n}\right)}\|g\|_{L^{q}\left(\mathbf{R}^{n}\right)}
$$

(ii) if $p=1$ or $q=1$ and $1 / p+1 / q=1 / r$,

$$
\|T(f, g)\|_{L^{1 / r, \infty}\left(\mathbf{R}^{n}\right)} \leq C\|f\|_{L^{p}\left(\mathbf{R}^{n}\right)}\|g\|_{L^{q}\left(\mathbf{R}^{n}\right)}
$$

(iii) if $1<p<\infty$, and $L_{c}^{\infty}\left(\mathbf{R}^{n}\right)$ denotes the class of compactly supported functions in $L^{\infty}\left(\mathbf{R}^{n}\right)$,

$$
\|T(f, g)\|_{L^{p}\left(\mathbf{R}^{n}\right)} \leq C\|f\|_{L^{p}\left(\mathbf{R}^{n}\right)}\|g\|_{L_{c}^{\infty}\left(\mathbf{R}^{n}\right)}
$$

and

$$
\|T(f, g)\|_{L^{p}\left(\mathbf{R}^{n}\right)} \leq C\|f\|_{L_{c}^{\infty}\left(\mathbf{R}^{n}\right)}\|g\|_{L^{p}\left(\mathbf{R}^{n}\right)}
$$

(iv) if $p=q=\infty$,

$$
\|T(f, g)\|_{B M O\left(\mathbf{R}^{n}\right)} \leq C\|f\|_{L^{\infty}\left(\mathbf{R}^{n}\right)}\|g\|_{L^{\infty}\left(\mathbf{R}^{n}\right)} .
$$

Remark 4.3 The boundedness estimate for $T: L^{p}\left(\mathbf{R}^{n}\right) \times L^{\infty}\left(\mathbf{R}^{n}\right) \rightarrow L^{p}\left(\mathbf{R}^{n}\right), 1<p<\infty$, clearly follows from the one for $T: L^{p}\left(\mathbf{R}^{n}\right) \times L^{p^{\prime}}\left(\mathbf{R}^{n}\right) \rightarrow L^{1}\left(\mathbf{R}^{n}\right), 1<p<\infty$ in part (i) and duality.

Remark 4.4 Another end-point result $T: L^{p}\left(\mathbf{R}^{n}\right) \times B M O\left(\mathbf{R}^{n}\right) \rightarrow L^{p}\left(\mathbf{R}^{n}\right), 1<p<\infty$ for paraproducts is also known. In fact, by freezing one function in $B M O$, the paraproducts can be realized as linear operators with Calderón-Zygmund kernels whose $L^{2}$ boundedness is obtained using Carleson measure estimates. From this the $L^{p} \times B M O \rightarrow L^{p}$ results follow by the linear Calderón-Zygmund theory for $1<p<\infty$, see [10]. We recover these estimates when the three families of molecules satisfy the cancellation condition (5) with $L=0$, see Theorem 4.9 below.

Remark 4.5 By realizing paraproducts as bilinear Calderón-Zygmund operators, rather than as linear ones, we can take advantage of the multilinear Calderón-Zygmund theory developed in [16], [17], [18], [6], [9], and [19]. As a consequence, some new paraproduct estimates such as the weighted and end-point estimates now follow in a unified way. In order to state them, let us fix some notation. The (uncentered) maximal function $M$ is defined on $f \in L_{l o c}^{1}\left(\mathbf{R}^{n}\right)$ by

$$
M(f)(x)=\sup _{Q \ni x} \frac{1}{|Q|} \int_{Q}|f(x)| d x
$$

where the sup runs over all cubes $Q \subset \mathbf{R}^{n}$ with sides parallel to the coordinate axes. Recall that a nonnegative weight $w \in L_{l o c}^{1}\left(\mathbf{R}^{n}\right)$ belongs to the $A_{p}$ Muckenhoupt class, for $1<p<\infty$, if there exists a constant $C$ such that

$$
\sup _{Q}\left(\frac{1}{|Q|} \int_{Q} w\right)\left(\frac{1}{|Q|} \int_{Q} w^{1-p^{\prime}}\right)^{p-1}<C
$$

where the cubes $Q$ are as above. We write $w \in A_{1}$ if there exists a constant $C$ such that $M(w)(x) \leq C w(x)$ for a.e. $x \in \mathbf{R}^{n}$ and set $A_{\infty}=\cup_{p \geq 1} A_{p}$. 
Theorem 4.6 (Paraproducts on weighted Lebesgue spaces) Let $\left\{\phi_{Q}^{1}\right\},\left\{\phi_{Q}^{2}\right\},\left\{\phi_{Q}^{3}\right\}$ be three families of molecules satisfying (3) with $N>10 n+10$ and $M=1$. Also, suppose that at least two of the families satisfy (5) with $L=0$. Let $T$ be the paraproduct

$$
T(f, g)=\sum_{Q \in \mathcal{D}}|Q|^{-1 / 2}\left\langle f, \phi_{Q}^{1}\right\rangle\left\langle g, \phi_{Q}^{2}\right\rangle \phi_{Q}^{3}, \quad f, g \in \mathcal{S}\left(\mathbf{R}^{n}\right) .
$$

(i) Then, if $1<p, q<\infty, r>0$, with $1 / p+1 / q=1 / r$, and $w$ is in $A_{\infty}$ then

$$
\|T(f, g)\|_{L^{r}\left(\mathbf{R}^{n}, w d x\right)} \leq C\|M(f)\|_{L^{p}\left(\mathbf{R}^{n}, w d x\right)}\|M(g)\|_{L^{q}\left(\mathbf{R}^{n}, w d x\right)} .
$$

In particular, if $w$ is in $A_{\min \{p, q\}}$, then

$$
\|T(f, g)\|_{L^{r}\left(\mathbf{R}^{n}, w d x\right)} \leq C\|f\|_{L^{p}\left(\mathbf{R}^{n}, w d x\right)}\|g\|_{L^{q}\left(\mathbf{R}^{n}, w d x\right)} .
$$

(ii) Also, if $w$ is in $A_{\infty}$, the following endpoint estimate holds

$$
\|T(f, g)\|_{L^{1 / 2, \infty}\left(\mathbf{R}^{n}, w d x\right)} \leq C\|M(f)\|_{L^{1}\left(\mathbf{R}^{n}, w d x\right)}\|M(g)\|_{L^{1}\left(\mathbf{R}^{n}, w d x\right)} .
$$

Hence, if $w$ is in $A_{1}$, we obtain

$$
w\left(\left\{x \in \mathbf{R}^{n}:|T(f, g)(x)|>\lambda\right\}\right) \leq \frac{C}{\lambda^{1 / 2}}\|f\|_{L^{1}\left(\mathbf{R}^{n}, w d x\right)}\|g\|_{L^{1}\left(\mathbf{R}^{n}, w d x\right)} .
$$

If more regularity on the molecules is assumed, one can derive boundedness results of the form $H^{p}\left(\mathbf{R}^{n}\right) \times$ $H^{q}\left(\mathbf{R}^{n}\right)$ into $L^{r}\left(\mathbf{R}^{n}\right)$ for $0<p, q, r<\infty$ and $1 / p+1 / q=1 / r$, since such results hold also for multilinear Calderón-Zygmund operators. Namely, we have

Theorem 4.7 (Paraproducts on Hardy spaces) Let $1<q_{1}, q_{2}, q<\infty$ such that $1 / q_{1}+1 / q_{2}=1 / q$, and let $0<p_{1}, p_{2}, p<\infty$ such that $1 / p_{1}+1 / p_{2}=1 / p$. Let $N_{0}$ be defined as follows:

(i) if $0<p_{1}, p_{2}, p \leq 1$, then $N_{0}=\lfloor n(1 / p-1)\rfloor$,

(ii) if one (but not both) of the $p_{j}^{\prime} s(j=1,2)$ is bigger than 1, then choose $\varepsilon$ and $\lambda$ such that $0<\varepsilon<$ $\min \left(1 / 2,1 / p_{1}, 1 / p_{2}\right)$ and $\lambda>\left(\min \left(1 / p_{1}, 1 / p_{2}\right)-\varepsilon\right)^{-1}$; and define $s$ by $1 / s=\lambda(1 / p-2 \varepsilon)+1 / p$. In this case, $N_{0}=\lfloor n(1 / s-1)\rfloor$.

Suppose that the three families of molecules $\left\{\phi_{Q}^{i}\right\}, i=1,2,3$ verify (3) with decay $N>10 n+5 N_{0}+5$, smoothness $M=N_{0}$, and at least two of the families satisfy (5) with $L=0$. Then the paraproduct (1) can be extended on products of Hardy spaces and it satisfies

$$
\|T(f, g)\|_{L^{p}\left(\mathbf{R}^{n}\right)} \leq C\|f\|_{H^{p_{1}\left(\mathbf{R}^{n}\right)}}\|g\|_{H^{p_{2}\left(\mathbf{R}^{n}\right)}},
$$

where $C$ depends only on $n, p_{1}, p_{2}, p, q_{1}, q_{2}, q$, and $N_{0}$.

Remark 4.8 Notice that the case $1<p_{1}, p_{2}<\infty$ in Theorem 4.7 is already covered by Theorem 4.2.

For a bilinear operator to reach values in a Hardy space, extra cancellation properties are required. We quantify this in the following result.

Theorem 4.9 (Paraproducts on homogeneous Triebel-Lizorkin spaces I) Let $0<p, q, r<\infty$ such that $1 / p+1 / q=1 / r$, and $1<s_{1}, s_{2} \leq \infty, 1 \leq s_{3}<\infty$ such that $1 / s_{1}+1 / s_{2}=1 / s_{3}$, and fix $N>n, t>0$ such that $t<\min (1, r)$ and set

$$
L=\lfloor 2 N / t-n\rfloor+1 \text {. }
$$

Suppose that the three families of molecules satisfy (3) with decay $5 N / t$, smoothness $L+1$, and (5) with cancellation $L$ as in (33). Then the paraproduct (1) satisfies

$$
\|T(f, g)\|_{\dot{F}_{r}^{0, s_{3}}\left(\mathbf{R}^{n}\right)} \leq C\|f\|_{\dot{F}_{p}^{0, s_{2}}\left(\mathbf{R}^{n}\right)}\|g\|_{\dot{F}_{q}^{0, s_{3}}\left(\mathbf{R}^{n}\right)} .
$$

In particular, since $\dot{F}_{p}^{0,2}=H^{p}$ for $0<p \leq 1$, and $\dot{F}_{p}^{0, a} \subset \dot{F}_{p}^{0, b}$ when $a<b$, T satisfies

$$
\|T(f, g)\|_{H^{r}\left(\mathbf{R}^{n}\right)} \leq C\|f\|_{H^{p}\left(\mathbf{R}^{n}\right)}\|g\|_{H^{q}\left(\mathbf{R}^{n}\right)} .
$$


Remark 4.10 As we mentioned earlier, under the conditions of Theorem 4.9, the bound for $T: H^{p}\left(\mathbf{R}^{n}\right) \times$ $H^{p^{\prime}}\left(\mathbf{R}^{n}\right) \rightarrow H^{1}\left(\mathbf{R}^{n}\right)$ gives, by duality, the bound for $T: L^{p}\left(\mathbf{R}^{n}\right) \times B M O\left(\mathbf{R}^{n}\right) \rightarrow L^{p}\left(\mathbf{R}^{n}\right), 1<p<\infty$.

For specific families of molecules we have a smoothing result

Theorem 4.11 (Special paraproducts on Sobolev spaces)

(i) Suppose that the three families of molecules $\left\{\psi_{P}^{1}\right\},\left\{\psi_{P}^{2}\right\}$, and $\left\{\psi_{P}^{3}\right\}$ are obtained by translations and dilations of functions $\psi^{1}, \psi^{2}, \psi^{3} \in B P F\left(\mathbf{R}^{n}\right)$. Then, for every $\alpha, \beta \in \mathbf{R}^{1}$ and $p, q, r$ with $1 / p+1 / q=1 / r$, $1<p, q<\infty$, there exists $C=C(\alpha, \beta, p, q, n)$ such that the bilinear paraproduct

$$
T(f, g)=\sum_{P \in \mathcal{D}}|P|^{-1 / 2}\left\langle f, \psi_{P}^{1}\right\rangle\left\langle g, \psi_{P}^{2}\right\rangle \psi_{P}^{3}
$$

satisfies

$$
\left\||\nabla|^{\alpha+\beta} T(f, g)\right\|_{L^{r}\left(\mathbf{R}^{n}\right)} \leq C\left\||\nabla|^{\alpha} f\right\|_{L^{p}\left(\mathbf{R}^{n}\right)}\left\||\nabla|^{\beta} g\right\|_{L^{q}\left(\mathbf{R}^{n}\right)}, \quad f, g \in \mathcal{S}_{0}\left(\mathbf{R}^{n}\right) .
$$

In particular, when $r \geq 1$ and $\alpha, \beta \geq 0, T$ is bounded from the product of Sobolev spaces $L_{\alpha}^{p}\left(\mathbf{R}^{n}\right) \times$ $L_{\beta}^{q}\left(\mathbf{R}^{n}\right)$ into $L_{\alpha+\beta}^{r}\left(\mathbf{R}^{n}\right)$.

(ii) If $\left\{\psi_{P}^{3}\right\}$ is replaced by a general family of molecules with $N=10 n+10, M=\alpha+\beta+1$ for some $\alpha, \beta \geq 0$ and no cancellation, then we still have

$$
\left\|\partial^{\kappa} T(f, g)\right\|_{L^{r}\left(\mathbf{R}^{n}\right)} \leq C\left\||\nabla|^{\alpha} f\right\|_{L^{p}\left(\mathbf{R}^{n}\right)}\left\||\nabla|^{\beta} g\right\|_{L^{q}\left(\mathbf{R}^{n}\right)}, \quad f, g \in \mathcal{S}_{0}\left(\mathbf{R}^{n}\right),
$$

for all multiindices $\kappa \in \mathbf{N}^{n}$ with $\alpha+\beta=|\kappa|$, and $1 / p+1 / q=1 / r, 1<p, q<\infty$.

The extremely simple proof of the next result illustrates the close interaction between the molecular decomposition of Triebel-Lizorkin spaces and the molecular representation of the paraproduct.

Theorem 4.12 (Paraproducts on Triebel-Lizorkin spaces II) Let $\alpha, \beta$ in $\mathbf{R}^{1}$. Suppose that $\left\{\phi_{P}^{1}\right\}$ is a family of dual molecules for $\dot{F}_{p}^{\alpha, s_{1}}\left(\mathbf{R}^{n}\right),\left\{\phi_{P}^{2}\right\}$ is a family of dual molecules for $\dot{F}_{q}^{\beta, s_{2}}\left(\mathbf{R}^{n}\right)$, and $\left\{\phi_{P}^{3}\right\}$ is a family of molecules for $\dot{F}_{r}^{\alpha+\beta, s_{3}}\left(\mathbf{R}^{n}\right)$, with $1 \leq p, q<\infty$ such that $1 / p+1 / q=1 / r$, and $1 \leq s_{1}, s_{2} \leq \infty$ such that $1 / s_{1}+1 / s_{2}=1 / s_{3}$ (and $s_{3}$ finite). Then $T$ verifies

$$
\|T(f, g)\|_{\dot{F}_{r}^{\alpha+\beta, s_{3}}\left(\mathbf{R}^{n}\right)} \leq C\|f\|_{\dot{F}_{p}^{\alpha, s_{1}}\left(\mathbf{R}^{n}\right)}\|g\|_{\dot{F}_{q}^{\beta, s_{2}}\left(\mathbf{R}^{n}\right)} .
$$

Remark 4.13 Theorems 4.9 and 4.12 can be used to obtain different versions of Theorems 4.2 (unweighted case) and Theorem 4.11; and Theorem 4.7, respectively, for general paraproducts. But we preferred to present the results for (unweighted) Lebesgue and Sobolev spaces in the way we did because of the independent interest in the arguments used. Also, combining Theorems 4.9 and 4.12 with Theorem 4.2, boundedness properties of paraproducts on inhomogeneous Triebel-Lizorkin spaces are easily derived.

Remark 4.14 Other end-point results for paraproducts of the form $\dot{F}_{p}^{\alpha, q} \times Y \rightarrow \dot{F}_{p}^{\alpha, q}$ for certain spaces $Y$ are given in [33] and [34].

Remark 4.15 Although norm equivalences for functions in several function spaces with the corresponding Triebel-Lizorkin norms are quite transparent, the actual identifications of the elements in the spaces requires further work because, strictly speaking, the homogeneous Triebel-Lizorkin spaces are spaces of distributions modulo polynomials. A detailed explanation of this issue and how to interpret expressions like $\left\langle f, \phi_{P}\right\rangle$ for distributions in certain Triebel-Lizorkin spaces and molecules without infinity vanishing moments is given in the Appendix B of [11]. The interested reader may consult [11] and the references therein for further details.

Remark 4.16 (Realization of paraproducts as pseudodifferential operators) It is a well-known fact by now that the study of Coifman-Meyer multipliers

$$
T_{m}(f, g)(x)=\int m(\xi, \eta) \widehat{f}(\xi) \widehat{g}(\eta) e^{i x \cdot(\xi+\eta)} d \xi d \eta,
$$


where

$$
\left|\partial_{\xi}^{\alpha} \partial_{\eta}^{\beta} m(\xi, \eta)\right| \leq C_{\alpha, \beta}(|\xi|+|\eta|)^{-|\alpha|-|\beta|},
$$

can be reduced to the study of paraproducts of the form (1) with at least two of the families of molecules involved having vanishing moments (but not necessarily the three of them). Conversely, it is not hard to prove that when the families of molecules are obtained by translations and dilations of functions in $B P F$, then the bilinear paraproducts generated by such families can be written as

$$
T(f, g)=\int m(x, \xi, \eta) \widehat{f}(\xi) \widehat{g}(\eta) e^{i x \cdot(\xi+\eta)} d \xi d \eta,
$$

where the symbol satisfies estimates of the form

$$
\left|\partial_{x}^{\rho} \partial_{\xi}^{\alpha} \partial_{\eta}^{\beta} m(x, \xi, \eta)\right| \leq C_{\rho, \alpha, \beta}(|\xi|+|\eta|)^{|\rho|-|\alpha|-|\beta|} .
$$

General pseudodifferential operators like the ones in (36) may not be bounded on Sobolev spaces and the special cancellations present in the families of molecules assumed in Theorem 4.11 seem to be crucial to obtain such boundedness results. For example, pseudodifferential operators with symbols satisfying the conditions

$$
\left|\partial_{x}^{\rho} \partial_{\xi}^{\alpha} \partial_{\eta}^{\beta} m(x, \xi, \eta)\right| \leq C_{\rho, \alpha, \beta}(1+|\xi|+|\eta|)^{|\rho|-|\alpha|-|\beta|} .
$$

where studied in [3] and it was shown there that in general they are not bounded on Lebesgue spaces and only map $L_{\alpha}^{p} \times L_{\alpha}^{q}$ into $L_{\alpha}^{r}$ for $\alpha>0$. This parallels the situation with the linear case and the so-called exotic or forbidden class of symbols $S_{1,1}^{0}$. See [3] for further details.

\section{Proofs of the boundedness results}

Proof of Theorem 4.1. We will prove that $T$ has a bilinear Calderón-Zygmund kernel. For this we do not need any cancellation at all in any of the families of molecules. The kernel $K(x, y, z)$ of $T$ is given by

$$
K(x, y, z)=\sum_{Q \in \mathcal{D}}|Q|^{-1 / 2} \phi_{Q}^{1}(y) \phi_{Q}^{2}(z) \phi_{Q}^{3}(x) .
$$

In order to show the size and regularity properties of $K(x, y, z)$ we will utilize the discrete almost orthogonality estimates proved in Section 3. By Proposition 3.6 with $\gamma=\nu=\mu=\lambda$ and Lemma 3.4 with $\beta=1$, the size estimate (15) for $K(x, y, z)$ becomes

$$
\begin{aligned}
|K(x, y, z)| & \leq \sum_{\nu \in \mathbf{Z}} \sum_{k \in \mathbf{Z}^{n}} 2^{n \nu / 2}\left|\phi_{\nu k}^{1}(y)\right|\left|\phi_{\nu k}^{2}(z)\right|\left|\phi_{\nu k}^{3}(x)\right| \\
& \leq \sum_{\nu \in \mathbf{Z}} \frac{C 2^{2 n \nu}}{\left[\left(1+2^{\nu}|x-y|\right)\left(1+2^{\nu}|z-y|\right)\left(1+2^{\nu}|x-z|\right)\right]^{N}} \\
& \leq \frac{C}{(|x-y|+|z-y|+|x-z|)^{2 n}} .
\end{aligned}
$$

Similarly, the regularity condition (16) with $\varepsilon=1$ follows from (3) with $M=1$. Indeed, Proposition 3.6 and Lemma 3.4 with $\beta=1+1 / n$ yield

$$
|\nabla K(x, y, z)| \leq \frac{C}{(|x-y|+|z-y|+|x-z|)^{2 n+1}} .
$$

Given $x, x^{\prime}, y$, and $z$ with $\left|x-x^{\prime}\right| \leq \frac{1}{2} \max \{|x-y|,|x-z|\}$ we get

$$
\left|K(x, y, z)-K\left(x^{\prime}, y, z\right)\right| \leq C \frac{\left|x-x^{\prime}\right|}{\left(\left|x^{\prime \prime}-y\right|+|z-y|+\left|x^{\prime \prime}-z\right|\right)^{2 n+1}}
$$


where $x^{\prime \prime}=t x+(1-t) x^{\prime}$ for some $t \in(0,1)$. Since

$$
\left|x^{\prime \prime}-y\right|=\left|x-y+t\left(x^{\prime}-x\right)\right| \geq|x-y|-t\left|x-x^{\prime}\right| \geq|x-y|-\frac{1}{2}|x-y|=\frac{1}{2}|x-y|,
$$

and, similarly, $\left|x^{\prime \prime}-z\right| \geq \frac{1}{2}|x-z|$, (16) follows. Notice that the case $\beta=1+1 / n$ now demands the condition $N>2 n+2$ (for Lemma 3.4 to apply) and therefore $5 N>10 n+10$. In order to prove the boundedness of $T$ on a product of Lebesgue spaces, we may assume (by taking transposes, if needed) that the families $\phi_{Q}^{1}$ and $\phi_{Q}^{2}$ are the ones with cancellation. The following argument is also well-known. Let $h$ be in $L^{\infty}$. Using the Cauchy-Schwartz inequality we obtain

$$
\begin{aligned}
|\langle T(f, g), h\rangle| & \leq \sum_{Q \in \mathcal{D}}\left|\left\langle f, \phi_{Q}^{1}\right\rangle\right|\left|\left\langle g, \phi_{Q}^{2}\right\rangle\right| 2^{n \nu / 2}\left|\left\langle h, \phi_{Q}^{3}\right\rangle\right| \\
& \leq\left(\sum_{Q \in \mathcal{D}}\left|\left\langle f, \phi_{Q}^{1}\right\rangle\right|^{2}\right)^{1 / 2}\left(\sum_{Q \in \mathcal{D}}\left|\left\langle g, \phi_{Q}^{2}\right\rangle\right|^{2}\right)^{1 / 2} \sup _{Q \in \mathcal{D}} 2^{n \nu / 2}\left|\left\langle h, \phi_{Q}^{3}\right\rangle\right| \\
& \leq C\|f\|_{L^{2}}\|g\|_{L^{2}}\|h\|_{L^{\infty}} .
\end{aligned}
$$

The quadratic estimates follow from Theorem 1 since, by Remark 2.1 part (i) and by Remark 2.2 with $p=s=2$ and $\alpha=0$, the first two families are also dual molecules for $L^{2}\left(\mathbf{R}^{n}\right)$. On the other hand,

$$
2^{n \nu / 2}\left|\left\langle h, \phi_{Q}^{3}\right\rangle\right| \leq C \int_{\mathbf{R}^{n}} \frac{2^{\nu n} h(x) d x}{\left(1+2^{\nu}\left|x-x_{Q}\right|\right)^{N}} \leq C\|h\|_{L^{\infty}\left(\mathbf{R}^{n}\right)} .
$$

Hence, $T$ maps $L^{2} \times L^{2}$ into $L^{1}$.

Proof of Theorem 4.2. Theorem 4.2 now follows from Theorem 4.1 and the already established theory of multilinear Calderón-Zygmund operators. In particular, we invoke the following result.

Theorem 5.1 (Grafakos-Torres, Theorems 1 and 3; Proposition 3 in [17]) Let $T$ be a bilinear CalderónZygmund operator. Then (i),(ii),(iii), and (iv) in Theorem 4.2 hold true for $T$.

The constants in Theorem 4.2 depend only on the constant of the bilinear Calderón-Zygmund kernel obtained in Theorem 4.1 and $\|T\|_{L^{2} \times L^{2} \rightarrow L^{1}}$.

Proof of Theorem 4.6. The proof is a direct consequence of the following result on bilinear Calderón-Zygmund operators

Theorem 5.2 (Grafakos-Torres, Corollary 3 and Remark 3 in [18]) Let $1<p, q<\infty, r>0$, with $1 / p+1 / q=$ $1 / r$ and $w \in A_{\infty}$. If $T$ is a bilinear Calderón-Zygmund operator, then

$$
\|T(f, g)\|_{L^{r}\left(\mathbf{R}^{n}, w d x\right)} \leq C\|M(f)\|_{L^{p}\left(\mathbf{R}^{n}, w d x\right)}\|M(g)\|_{L^{q}\left(\mathbf{R}^{n}, w d x\right)},
$$

and

$$
\|T(f, g)\|_{L^{1 / 2, \infty}\left(\mathbf{R}^{n}, w d x\right)} \leq C\|M(f)\|_{L^{1}\left(\mathbf{R}^{n}, w d x\right)}\|M(g)\|_{L^{1}\left(\mathbf{R}^{n}, w d x\right)},
$$

where $C$ depends only on $p, n,\|T\|_{L^{1} \times L^{1} \rightarrow L^{1 / 2, \infty}}$, and the size and smoothness constant of the kernel of $T$.

Proof of Theorem 4.7. The proof is once again based on the fact that paraproducts can be realized as bilinear Calderón-Zygmund operators. Let $1<q_{1}, q_{2}, q<\infty$ such that $1 / q_{1}+1 / q_{2}=1 / q$, let $0<p_{1}, p_{2}, p<\infty$ such that $1 / p_{1}+1 / p_{2}=1 / p$, and take $N_{0}$ as in Theorem 4.7. With these parameters, we now use the following theorem.

Theorem 5.3 (Grafakos-Kalton, Theorem 1.1 in [15]) Suppose that is $T$ be a bilinear Calderón-Zygmund operator whose kernel $K(x, y, z)$ satisfies

$$
\left|\partial_{x}^{\alpha_{1}} \partial_{y}^{\alpha_{2}} \partial_{z}^{\alpha_{3}} K(x, y, z)\right| \leq \frac{C_{\alpha}}{(|x-y|+|y-z|+|x-z|)^{2 n+|\alpha|}}, \quad \text { for all }|\alpha| \leq N_{0},
$$


where the triples $\alpha=\left(\alpha_{1}, \alpha_{2}, \alpha_{3}\right)$ consist of nonnegative integers and $|\alpha|=\alpha_{1}+\alpha_{2}+\alpha_{3}$. Also, suppose that $T$ admits an extension that maps $L^{q_{1}}\left(\mathbf{R}^{n}\right) \times L^{q_{2}}\left(\mathbf{R}^{n}\right)$ into $L^{q}\left(\mathbf{R}^{n}\right)$, then

$$
\|T(f, g)\|_{L^{p}\left(\mathbf{R}^{n}\right)} \leq C\|f\|_{H^{p_{1}\left(\mathbf{R}^{n}\right)}}\|g\|_{H^{p_{2}\left(\mathbf{R}^{n}\right)}},
$$

where $C$ depends only on $n, p_{1}, p_{2}, p, q_{1}, q_{2}, q, \sup _{\alpha:|\alpha| \leq N_{0}} C_{\alpha}$ and $\|T\|_{L^{q_{1}} \times L^{q_{2}} \rightarrow L^{q}}$.

The decay, smoothness, and cancellation conditions of the molecules imply (by Theorem 4.1) that $T$ is a bilinear Calderón-Zygmund operator. In order to check (38) one can proceed as in the proof of Theorem 4.1. In this case, use Proposition 3.6 with $\gamma=\lambda=\nu=\mu$ and Lemma 3.4 with $\beta=|\alpha| / n+1$. Notice that, by hypothesis, $N>10 n+5 N_{0}+5$, so that $N / 5>n+n\left(N_{0} / n+1\right)+1 \geq n+n \beta+1$ and the conditions in Lemma 3.4 are met.

Proof of Theorem 4.9. In order to prove Theorem 4.9 we do not use the realization of the paraproduct as a bilinear Calderón-Zygmund operator. Instead, we will use the following bilinear almost diagonal estimate.

Theorem 5.4 (Grafakos-Torres, Theorem 4 in [16]) Let $0<p, q, r<\infty$ such that $1 / p+1 / q=1 / r$, and $1<s_{1}, s_{2} \leq \infty, 1 \leq s_{3}<\infty$ such that $1 / s_{1}+1 / s_{2}=1 / s_{3}$, and fix $t<\min (1, r)$. Take $\psi \in B P F\left(\mathbf{R}^{n}\right)$ and consider its translations and dilations $\psi_{\nu k}(x)=2^{\nu n / 2} \psi\left(2^{\nu} x-k\right)$. Assume that a bilinear operator $T$ verifies

$$
\begin{aligned}
& \left|\left\langle T\left(\psi_{\nu k}, \psi_{\mu l}\right), \psi_{\lambda m}\right\rangle\right| \leq \\
& \frac{C 2^{-(\max (\mu, \nu, \lambda)-\min (\mu, \nu, \lambda)) \varepsilon / t} 2^{(-\max (\mu, \nu, \lambda)+\operatorname{med}(\mu, \nu, \lambda)+\min (\mu, \nu, \lambda)) n / 2 t} 2^{(-\nu-\mu+\lambda) n(1-t) / 2 t}}{\left(\left(1+2^{\min (\nu, \mu)}\left|2^{-\nu} k-2^{-\mu} l\right|\right)\left(1+2^{\min (\mu, \lambda)}\left|2^{-\mu} l-2^{-\lambda} m\right|\right)\left(1+2^{\min (\lambda, \nu)}\left|2^{-\lambda} m-2^{-\nu} k\right|\right)\right)^{N / t}}
\end{aligned}
$$

for some $C>0, N>n$, and $\varepsilon>0$. Then the operator $T$ can be extended to be a bounded operator from $\dot{F}_{p}^{0, s_{1}}\left(\mathbf{R}^{n}\right) \times \dot{F}_{q}^{0, s_{2}}\left(\mathbf{R}^{n}\right)$ into $\dot{F}_{r}^{0, s_{3}}\left(\mathbf{R}^{n}\right)$.

Remark 5.5 There is a typo in the statement of Theorem 4 in [16]. The factor on the right hand side should read $2^{(-\nu-\mu+\lambda) n(1-t) / 2 t}$ as shown here, and not $2^{(-\nu-\mu+\lambda) n(t-1) / 2 t}$ as it appears in [16].

Theorem 4.9 will then follow from Theorem 5.4 once we show that

$$
\begin{aligned}
& \sum_{Q \in \mathcal{D}} 2^{n \nu / 2}\left|\left\langle\psi_{\nu k}, \phi_{Q}^{1}\right\rangle\right|\left|\left\langle\psi_{\mu l}, \phi_{Q}^{2}\right\rangle\right|\left|\left\langle\psi_{\lambda m}, \phi_{Q}^{3}\right\rangle\right| \\
& \leq \frac{C 2^{-(\max (\mu, \nu, \lambda)-\min (\mu, \nu, \lambda)) \varepsilon / t} 2^{(-\max (\mu, \nu, \lambda)+\operatorname{med}(\mu, \nu, \lambda)+\min (\mu, \nu, \lambda)) n / 2 t} 2^{(-\nu-\mu+\lambda) n(1-t) / 2 t}}{\left(\left(1+2^{\min (\nu, \mu)}\left|2^{-\nu} k-2^{-\mu} l\right|\right)\left(1+2^{\min (\mu, \lambda)}\left|2^{-\mu} l-2^{-\lambda} m\right|\right)\left(1+2^{\min (\lambda, \nu)}\left|2^{-\lambda} m-2^{-\nu} k\right|\right)\right)^{N / t}}
\end{aligned}
$$

Notice that, except for the factor $2^{(-\nu-\mu+\lambda) n(1-t) / 2 t}$, the right hand side is symmetric on $\mu, \nu$, and $\lambda$. Since we are assuming the same decay, smoothness, and cancellation conditions on the three families of molecules $\left\{\phi_{Q}^{i}\right\}, i=1,2,3$, it is enough to consider only the case $\lambda \leq \mu \leq \nu$ (as this case minimizes the factor $\left.2^{(-\nu-\mu+\lambda) n(1-t) / 2 t}\right)$. Hence, (39) reduces to

$$
\begin{aligned}
& \sum_{Q \in \mathcal{D}} 2^{n \nu / 2}\left|\left\langle\psi_{\nu k}, \phi_{Q}^{1}\right\rangle\right|\left|\left\langle\psi_{\mu l}, \phi_{Q}^{2}\right\rangle\right|\left|\left\langle\psi_{\lambda m}, \phi_{Q}^{3}\right\rangle\right| \\
& \leq \frac{C 2^{-(\nu-\lambda)(\varepsilon+n) / t} 2^{\mu n / 2+\nu n / 2-\lambda n / 2}}{\left(\left(1+2^{\mu}\left|2^{-\nu} k-2^{-\mu} l\right|\right)\left(1+2^{\lambda}\left|2^{-\mu} l-2^{-\lambda} m\right|\right)\left(1+2^{\lambda}\left|2^{-\lambda} m-2^{-\nu} k\right|\right)\right)^{N / t}}
\end{aligned}
$$

The molecules $\psi_{\nu k}$ have arbitrary decay, smoothness, and cancellation. Also, for $L$ as in (33) we clearly have $5 N / t>L+n+1$. We are now in position to apply Proposition 3.2 with $N_{1}=N_{2}=5 N / t>n$ and Proposition 


\section{6 to obtain}

$$
\begin{aligned}
& \sum_{Q=Q_{j \gamma} \in \mathcal{D}} 2^{n \nu / 2}\left|\left\langle\psi_{\nu k}, \phi_{Q}^{1}\right\rangle\right|\left|\left\langle\psi_{\mu l}, \phi_{Q}^{2}\right\rangle\right|\left|\left\langle\psi_{\lambda m}, \phi_{Q}^{3}\right\rangle\right| \\
\leq & \sum_{\substack{j \in \mathbf{Z}^{n} \\
\gamma \in \mathbf{Z}}} \frac{C 2^{\gamma n / 2} 2^{-|\nu-\gamma|(L+1+n / 2)} 2^{-|\mu-\gamma|(L+1+n / 2)} 2^{-|\lambda-\gamma|(L+1+n / 2)}}{\left(\left(1+2^{\min (\nu, \gamma)}\left|x_{\nu}-2^{-\gamma} j\right|\right)\left(1+2^{\min (\mu, \gamma)}\left|x_{\mu}-2^{-\gamma} j\right|\right)\left(1+2^{(\min (\lambda, \gamma)}\left|x_{\lambda}-2^{-\gamma} j\right|\right)\right)^{5 N / t}} \\
= & \sum_{\gamma \in \mathbf{Z}} C 2^{3 n \gamma / 2} 2^{-(|\nu-\gamma|+|\mu-\gamma|+|\lambda-\gamma|)(L+1+n / 2)} 2^{-(\min (\nu, \gamma)+\min (\mu, \gamma)+\min (\lambda, \gamma)) n / 2} \\
\times & \sum_{j \in \mathbf{Z}^{n}} \frac{2^{-\gamma n} 2^{\min (\nu, \gamma) n / 2} 2^{\min (\mu, \gamma) n / 2} 2^{\min (\lambda, \gamma) n / 2}}{\left(\left(1+2^{\min (\nu, \gamma)}\left|x_{\nu}-2^{-\gamma} j\right|\right)\left(1+2^{\min (\mu, \gamma)}\left|x_{\mu}-2^{-\gamma} j\right|\right)\left(1+2^{(\min (\lambda, \gamma)}\left|x_{\lambda}-2^{-\gamma} j\right|\right)\right)^{5 N / t}} \\
\leq & C \sum_{\gamma \in \mathbf{Z}} 2^{3 n \gamma / 2} 2^{-(|\nu-\gamma|+|\mu-\gamma|+|\lambda-\gamma|)(L+1+n / 2)} 2^{-(\min (\nu, \gamma)+\min (\mu, \gamma)+\min (\lambda, \gamma)) n / 2} \\
\times & \frac{2^{(-\max (\min (\nu, \gamma), \min (\mu, \gamma), \min (\lambda, \gamma))+\operatorname{med}(\min (\nu, \gamma), \min (\mu, \gamma), \min (\lambda, \gamma))+\min (\nu, \mu, \lambda, \gamma)) n / 2}}{\left[\left(1+2^{\min (\nu, \gamma, \mu)}\left|x_{\nu}-x_{\mu}\right|\right)\left(1+2^{\min (\mu, \gamma, \lambda)}\left|x_{\mu}-x_{\lambda}\right|\right)\left(1+2^{\min (\nu, \gamma, \lambda)}\left|x_{\nu}-x_{\lambda}\right|\right)\right]^{N / t}} \\
= & \sum_{\gamma \in \mathbf{Z}} \frac{C 2^{p(\nu, \mu, \lambda, \gamma)}}{\left[\left(1+2^{\min (\nu, \gamma, \mu)}\left|x_{\nu}-x_{\mu}\right|\right)\left(1+2^{\min (\mu, \gamma, \lambda)}\left|x_{\mu}-x_{\lambda}\right|\right)\left(1+2^{\min (\nu, \gamma, \lambda)}\left|x_{\nu}-x_{\lambda}\right|\right)\right]^{N / t}}
\end{aligned}
$$

where $x_{\nu}=2^{-\nu} k, x_{\mu}=2^{-\mu} l$; and $x_{\lambda}=2^{-\lambda} m$, and the power $p(\nu, \mu, \lambda, \gamma)$ is given by

$$
\begin{aligned}
p(\nu, \mu, \lambda, \gamma) & =3 n \gamma / 2-(|\nu-\gamma|+|\mu-\gamma|+|\lambda-\gamma|)(L+1+n / 2) \\
& -(\min (\nu, \gamma)+\min (\mu, \gamma)+\min (\lambda, \gamma)) n / 2 \\
& -\max (\min (\nu, \gamma), \min (\mu, \gamma), \min (\lambda, \gamma)) n / 2 \\
& +\operatorname{med}(\min (\nu, \gamma), \min (\mu, \gamma), \min (\lambda, \gamma)) n / 2 \\
& +\min (\nu, \mu, \lambda, \gamma) n / 2 .
\end{aligned}
$$

At this point we split the sum in $\gamma \in \mathbf{Z}$ into four sums according to the following cases.

Case 1: $\gamma \geq \nu \geq \mu \geq \lambda$. This case gives

$$
\begin{aligned}
& \sum_{\substack{\gamma \in \mathbf{Z} \\
\gamma \geq \nu}} \frac{C 2^{p(\nu, \mu, \lambda, \gamma)}}{\left[\left(1+2^{\min (\nu, \gamma, \mu)}\left|x_{\nu}-x_{\mu}\right|\right)\left(1+2^{\min (\mu, \gamma, \lambda)}\left|x_{\mu}-x_{\lambda}\right|\right)\left(1+2^{\min (\nu, \gamma, \lambda)}\left|x_{\nu}-x_{\lambda}\right|\right)\right]^{N / t}} \\
& =C \sum_{\substack{\gamma \in \mathbf{Z} \\
\gamma \geq \nu}} \frac{2^{-(L+1)((\gamma-\nu)+(\gamma-\mu)+(\gamma-\lambda))+\lambda n / 2+\mu n / 2-\nu n / 2}}{\left[\left(1+2^{\mu}\left|x_{\nu}-x_{\mu}\right|\right)\left(1+2^{\lambda}\left|x_{\mu}-x_{\lambda}\right|\right)\left(1+2^{\lambda}\left|x_{\nu}-x_{\lambda}\right|\right)\right]^{N / t}} \\
& \leq \frac{C 2^{-\lambda n / 2+\mu n / 2+\nu n / 2}}{\left[\left(1+2^{\mu}\left|x_{\nu}-x_{\mu}\right|\right)\left(1+2^{\lambda}\left|x_{\mu}-x_{\lambda}\right|\right)\left(1+2^{\lambda}\left|x_{\nu}-x_{\lambda}\right|\right)\right]^{N / t}} \sum_{\substack{\gamma \in \mathbf{Z} \\
\gamma \geq \nu}} 2^{-(L+1)(\gamma-\lambda)} \\
& \leq \frac{C 2^{-\lambda n / 2+\mu n / 2+\nu n / 2} 2^{-(L+1)(\nu-\lambda)}}{\left[\left(1+2^{\mu}\left|x_{\nu}-x_{\mu}\right|\right)\left(1+2^{\lambda}\left|x_{\mu}-x_{\lambda}\right|\right)\left(1+2^{\lambda}\left|x_{\nu}-x_{\lambda}\right|\right)\right]^{N / t}},
\end{aligned}
$$

where $C$ depends only on $N, n$ and $L$. 
Case 2: $\nu \geq \gamma \geq \mu \geq \lambda$. This case gives

$$
\begin{aligned}
& \sum_{\substack{\gamma \in \mathbf{Z} \\
\nu \geq \gamma \geq \mu}} \frac{C 2^{p(\nu, \mu, \lambda, \gamma)}}{\left[\left(1+2^{\min (\nu, \gamma, \mu)}\left|x_{\nu}-x_{\mu}\right|\right)\left(1+2^{\min (\mu, \gamma, \lambda)}\left|x_{\mu}-x_{\lambda}\right|\right)\left(1+2^{\min (\nu, \gamma, \lambda)}\left|x_{\nu}-x_{\lambda}\right|\right)\right]^{N / t}} \\
= & C \sum_{\substack{\gamma \in \mathbf{Z} \\
\nu \geq \gamma \geq \mu}} \frac{2^{-(L+1)(\nu-\gamma)-(L+1)(\gamma-\mu)-(L+1)(\gamma-\lambda)+(-\nu+\mu+\lambda) n / 2}}{\left[\left(1+2^{\mu}\left|x_{\nu}-x_{\mu}\right|\right)\left(1+2^{\lambda}\left|x_{\mu}-x_{\lambda}\right|\right)\left(1+2^{\lambda}\left|x_{\nu}-x_{\lambda}\right|\right)\right]^{N / t}} \\
\leq & \frac{C 2^{-\nu n / 2+\mu n / 2+\lambda n / 2} 2^{-(\nu-\mu)(L+1)}}{\left[\left(1+2^{\mu}\left|x_{\nu}-x_{\mu}\right|\right)\left(1+2^{\lambda}\left|x_{\mu}-x_{\lambda}\right|\right)\left(1+2^{\lambda}\left|x_{\nu}-x_{\lambda}\right|\right)\right]^{N / t}} \sum_{\gamma \in \mathbf{Z}} 2^{-(L+1)(\gamma-\lambda)} \\
\leq & \frac{C 2^{-\lambda n / 2+\mu n / 2+\nu n / 2} 2^{-(L+1)(\nu-\lambda)}}{\left[\left(1+2^{\mu}\left|x_{\nu}-x_{\mu}\right|\right)\left(1+2^{\lambda}\left|x_{\mu}-x_{\lambda}\right|\right)\left(1+2^{\lambda}\left|x_{\nu}-x_{\lambda}\right|\right)\right]^{N / t}},
\end{aligned}
$$

where $C$ depends only on $N, n$ and $L$.

Case 3: $\nu \geq \mu \geq \gamma \geq \lambda$. In this case we have

$$
\begin{aligned}
& \sum_{\substack{\gamma \in \mathbf{Z} \\
\mu \geq \gamma \geq \lambda}} \frac{C 2^{p(\nu, \mu, \lambda, \gamma)}}{\left[\left(1+2^{\min (\nu, \gamma, \mu)}\left|x_{\nu}-x_{\mu}\right|\right)\left(1+2^{\min (\mu, \gamma, \lambda)}\left|x_{\mu}-x_{\lambda}\right|\right)\left(1+2^{\min (\nu, \gamma, \lambda)}\left|x_{\nu}-x_{\lambda}\right|\right)\right]^{N / t}} \\
= & C \sum_{\substack{\gamma \in \mathbf{Z} \\
\mu \geq \gamma \geq \lambda}} \frac{2^{-(\nu-\lambda)(L+1)-\lambda n / 2+\mu n / 2+\nu n / 2-(\mu-\gamma)(L+1+n)-(\nu-\lambda) n}}{\left[\left(1+2^{\gamma}\left|x_{\nu}-x_{\mu}\right|\right)\left(1+2^{\lambda}\left|x_{\mu}-x_{\lambda}\right|\right)\left(1+2^{\lambda}\left|x_{\nu}-x_{\lambda}\right|\right)\right]^{N / t}} \\
\leq & \frac{C 2^{-(\nu-\lambda)(L+1)-\lambda n / 2+\mu n / 2+\nu n / 2}}{\left[\left(1+2^{\lambda}\left|x_{\mu}-x_{\lambda}\right|\right)\left(1+2^{\lambda}\left|x_{\nu}-x_{\lambda}\right|\right)\right]^{N / t}} \sum_{\substack{\gamma \in \mathbf{Z} \\
\mu \geq \gamma \geq \lambda}} \frac{2^{-(\mu-\gamma)(L+1+n)}}{\left(1+2^{\gamma}\left|x_{\nu}-x_{\mu}\right|\right)^{N / t}} \\
\leq & \frac{C 2^{-(\nu-\lambda)(L+1)-\lambda n / 2+\mu n / 2+\nu n / 2}}{\left[\left(1+2^{\lambda}\left|x_{\mu}-x_{\lambda}\right|\right)\left(1+2^{\lambda}\left|x_{\nu}-x_{\lambda}\right|\right)\right]^{N / t}} \sum_{\substack{\gamma \in \mathbf{Z} \\
\mu \geq \gamma \geq \lambda}} \frac{2^{-(\mu-\gamma)(L+1+n)}}{\left(2^{\gamma-\mu}+2^{\gamma-\mu+\mu}\left|x_{\nu}-x_{\mu}\right|\right)^{N / t}} \\
\leq & \frac{C 2^{-(\nu-\lambda)(L+1)-\lambda n / 2+\mu n / 2+\nu n / 2}}{\left[\left(1+2^{\lambda}\left|x_{\mu}-x_{\lambda}\right|\right)\left(1+2^{\lambda}\left|x_{\nu}-x_{\lambda}\right|\right)\left(1+2^{\mu}\left|x_{\nu}-x_{\mu}\right|\right)\right]^{N / t}} \sum_{\substack{\gamma \in \mathbf{Z} \\
\mu \geq \gamma}} 2^{-(\mu-\gamma)(L+1+n-N / t)}
\end{aligned}
$$

and the last sum converges to a number depending only on $N, n, L$, and $t$, provided that $L+1+n-N / t>0$. 
Case 4: $\nu \geq \mu \geq \lambda \geq \gamma$. In this case we have

$$
\begin{aligned}
& \sum_{\substack{\gamma \in \mathbf{Z} \\
\gamma \leq \lambda}} \frac{C 2^{p(\nu, \mu, \lambda, \gamma)}}{\left[\left(1+2^{\min (\nu, \gamma, \mu)}\left|x_{\nu}-x_{\mu}\right|\right)\left(1+2^{\min (\mu, \gamma, \lambda)}\left|x_{\mu}-x_{\lambda}\right|\right)\left(1+2^{\min (\nu, \gamma, \lambda)}\left|x_{\nu}-x_{\lambda}\right|\right)\right]^{N / t}} \\
& =C \sum_{\substack{\gamma \in \mathbf{Z} \\
\gamma \leq \lambda}} \frac{2^{-(\nu-\lambda)(L+1)-\lambda n / 2+\mu n / 2+\nu n / 2-(\nu-\gamma)(L+1)-(\mu-\gamma)(L+1+n)-(\lambda-\gamma)(L+1+n)}}{\left[\left(1+2^{\gamma}\left|x_{\nu}-x_{\mu}\right|\right)\left(1+2^{\gamma}\left|x_{\mu}-x_{\lambda}\right|\right)\left(1+2^{\gamma}\left|x_{\nu}-x_{\lambda}\right|\right)\right]^{N / t}} \\
& \leq C \sum_{\substack{\gamma \in \mathbf{Z} \\
\gamma \leq \lambda}} \frac{2^{-(\nu-\lambda)(L+1)-\lambda n / 2+\mu n / 2+\nu n / 2-(\mu-\gamma)(L+1+n)-(\lambda-\gamma)(L+1+n)}}{\left[\left(2^{\gamma-\mu}+2^{\gamma}\left|x_{\nu}-x_{\mu}\right|\right)\left(2^{\gamma-\lambda}+2^{\gamma}\left|x_{\mu}-x_{\lambda}\right|\right)\left(2^{\gamma-\lambda}+2^{\gamma}\left|x_{\nu}-x_{\lambda}\right|\right)\right]^{N / t}} \\
& =C \sum_{\substack{\gamma \in \mathbf{Z} \\
\gamma \leq \lambda}} \frac{2^{-(\nu-\lambda)(L+1)-\lambda n / 2+\mu n / 2+\nu n / 2-(\mu-\gamma)(L+1+n-N / t)-(\lambda-\gamma)(L+1+n-2 N / t)}}{\left[\left(1+2^{\mu}\left|x_{\nu}-x_{\mu}\right|\right)\left(1+2^{\lambda}\left|x_{\mu}-x_{\lambda}\right|\right)\left(1+2^{\lambda}\left|x_{\nu}-x_{\lambda}\right|\right)\right]^{N / t}} \\
& \leq \frac{2^{-(\nu-\lambda)(L+1)-\lambda n / 2+\mu n / 2+\nu n / 2}}{\left[\left(1+2^{\mu}\left|x_{\nu}-x_{\mu}\right|\right)\left(1+2^{\lambda}\left|x_{\mu}-x_{\lambda}\right|\right)\left(1+2^{\lambda}\left|x_{\nu}-x_{\lambda}\right|\right)\right]^{N / t}} \sum_{\substack{\gamma \in \mathbf{Z} \\
\gamma \leq \lambda}} 2^{-(\lambda-\gamma)(L+1+n-2 N / t)},
\end{aligned}
$$

and the last sum converges to a number depending only on $N, n, L$, and $t$, provided that $L+1+n-2 N / t>0$. Thus, we have proved

$$
\begin{aligned}
& \sum_{Q \in \mathcal{D}} 2^{n \nu / 2}\left|\left\langle\psi_{\nu k}, \phi_{Q}^{1}\right\rangle\right|\left|\left\langle\psi_{\mu l}, \phi_{Q}^{2}\right\rangle\right|\left|\left\langle\psi_{\lambda m}, \phi_{Q}^{3}\right\rangle\right| \\
& \leq C \frac{2^{-(\nu-\lambda)(L+1)-\lambda n / 2+\mu n / 2+\nu n / 2}}{\left[\left(1+2^{\mu}\left|x_{\nu}-x_{\mu}\right|\right)\left(1+2^{\lambda}\left|x_{\mu}-x_{\lambda}\right|\right)\left(1+2^{\lambda}\left|x_{\nu}-x_{\lambda}\right|\right)\right]^{N / t}} .
\end{aligned}
$$

Consequently, since $L+1+n>2 N / t$ and $N>n$ we obtain $L+1>n(1-t) / t+n / t$ and (40) follows from (41) with $\varepsilon=n(1-t)$.

Proof Theorem 4.11. Given $\alpha, \beta \in \mathbf{R}^{1}$ set $l=(-\alpha,-\beta, \alpha+\beta)$. For Schwartz functions $\psi^{1}, \psi^{2}, \psi^{3} \in$ $\operatorname{BPF}\left(\mathbf{R}^{n}\right)$ define Schwartz functions $\Psi^{1}, \Psi^{2}, \Psi^{3}$ in terms of their Fourier transforms by

$$
\widehat{\Psi^{j}}(\xi)=|\xi|^{l_{j}} \widehat{\psi^{j}}(\xi), \quad \xi \in \mathbf{R}^{n}, j=1,2,3 ;
$$

where the right-hand side is well defined even for $l_{j}<0$, since $\widehat{\Psi^{j}}$ vanishes in a neighborhood of $\xi=0$. In fact, we have $\operatorname{supp}\left(\widehat{\Psi^{j}}\right)=\operatorname{supp}\left(\widehat{\psi^{j}}\right)$, and for each dyadic cube $P=P_{\nu k}$,

$$
\widehat{\Psi_{P}^{j}}(\xi)=|P|^{l_{j} / n}|\xi|^{l_{j}} \widehat{\psi_{P}^{j}}(\xi), \quad \xi \in \mathbf{R}^{n},
$$

where $\psi_{P}^{j}(x)=2^{\nu n / 2} \psi^{j}\left(2^{\nu} x-k\right)$ and $\Psi_{P}^{j}=2^{\nu n / 2} \Psi^{j}\left(2^{\nu} x-k\right)$. Consequently, $\Psi_{P}^{j}$ is a smooth molecule associated to $P$ with arbitrary decay and cancellation. Plancherel's identity and (42) allow to write, for each $f, g$ in $\mathcal{S}_{0}$,

$$
\begin{aligned}
|\nabla|^{(\alpha+\beta)} T(f, g) & =\sum_{P \in \mathcal{D}}|P|^{-1 / 2}\left\langle f, \psi_{P}^{1}\right\rangle\left\langle g, \psi_{P}^{2}\right\rangle|\nabla|^{(\alpha+\beta)} \psi_{P}^{3} \\
& =\sum_{P \in \mathcal{D}}|P|^{-1 / 2}\left\langle\widehat{f},|P|^{\alpha / n}|\xi|^{\alpha} \widehat{\Psi_{P}^{1}}\right\rangle\left\langle\widehat{g},|P|^{\beta / n}|\xi|^{\beta} \widehat{\Psi_{P}^{2}}\right\rangle|P|^{-(\alpha+\beta) / n} \Psi_{P}^{3} \\
& =\sum_{P \in \mathcal{D}}|P|^{-1 / 2}\left\langle|\nabla|^{\alpha} f, \Psi_{P}^{1}\right\rangle\left\langle|\nabla|^{\beta} g, \Psi_{P}^{2}\right\rangle \Psi_{P}^{3} \\
& =\mathcal{T}\left(|\nabla|^{\alpha} f,|\nabla|^{\beta} g\right),
\end{aligned}
$$


where the paraproduct $\mathcal{T}$ associated to the families $\left\{\Psi_{P}^{j}\right\}_{j=1,2,3}$ verifies the conditions of Theorem 4.2, which yields (34). In order to prove (35) we only need to slightly change the proof of (34). Given an arbitrary family of molecules $\left\{\psi_{P}^{3}\right\}$ as in the statement, now set $\Psi_{P}^{3}=|P|^{(\alpha+\beta) / n} \partial^{\kappa} \psi_{P}^{3}$. By (3), the molecule $\Psi_{P}^{3}$ has decay $N>10 n+10$ (as $\psi_{P}^{3}$ does) and smoothness $\alpha+\beta+1-|\kappa|=1$. The proof of (35) now follows from the one for (34) applied to $\Psi_{P}^{3}$.

Proof Theorem 4.12. Set

$$
h=T(f, g)=\sum_{Q \in \mathcal{D}}|Q|^{-1 / 2}\left\langle f, \phi_{Q}^{1}\right\rangle\left\langle g, \phi_{Q}^{2}\right\rangle \phi_{Q}^{3}
$$

and $c_{Q}=|Q|^{-1 / 2}\left\langle f, \phi_{Q}^{1}\right\rangle\left\langle g, \phi_{Q}^{2}\right\rangle$, so that $h=\sum_{Q} c_{Q} \phi_{Q}^{3}$. By (12) we have

$$
\|h\|_{\dot{F}_{r}^{\alpha+\beta, s_{3}}} \leq C\left\|\left\{c_{Q}\right\}\right\|_{\dot{f}_{r}^{\alpha+\beta, s_{3}}} .
$$

Then, an iteration of Hölder's inequality yields

$$
\begin{aligned}
& \|T(f, g)\|_{\dot{F}_{r}^{\alpha+\beta, s_{3}}}=\|h\|_{\dot{F}_{r}^{\alpha+\beta, s_{3}}} \leq C\left\|\left\{c_{Q}\right\}\right\|_{\dot{f}_{r}^{\alpha+\beta, s_{3}}} \\
& =C\left\|\left[\sum_{Q=Q(\nu, k) \in \mathcal{D}}\left(|Q|^{-1 / 2}\left|\left\langle f, \phi_{Q}^{1}\right\rangle\right|\left|\left\langle g, \phi_{Q}^{2}\right\rangle\right| 2^{(\alpha+\beta+n / 2) \nu} \chi_{Q}\right)^{s_{3}}\right]^{1 / s_{3}}\right\|_{L^{r}} \\
& =C\left\|\left[\sum_{\nu, k}\left(2^{(\alpha+n / 2) \nu}\left|\left\langle f, \phi_{Q}^{1}\right\rangle\right| \chi_{Q}\left|\left\langle g, \phi_{Q}^{2}\right\rangle\right| 2^{(\beta+n / 2) \nu} \chi_{Q}\right)^{s_{3}}\right]^{1 / s_{3}}\right\|_{L^{r}} \\
& \leq C\left\|\left[\sum_{\nu, k}\left(2^{(\alpha+n / 2) \nu}\left|\left\langle f, \phi_{Q}^{1}\right\rangle\right| \chi_{Q}\right)^{s_{1}}\right]^{1 / s_{1}}\left[\sum_{\nu, k}\left(2^{(\beta+n / 2) \nu}\left|\left\langle g, \phi_{Q}^{2}\right\rangle\right| \chi_{Q}\right)^{s_{2}}\right]^{1 / s_{2}}\right\|_{L^{r}} \\
& \leq C\left\|\left[\sum_{\nu, k}\left(2^{(\alpha+n / 2) \nu}\left|\left\langle f, \phi_{Q}^{1}\right\rangle\right| \chi_{Q}\right)^{s_{1}}\right]^{1 / s_{1}}\right\|\left\|_{L^{p}}\right\|\left[\sum_{\nu, k}\left(2^{(\beta+n / 2) \nu}\left|\left\langle g, \phi_{Q}^{2}\right\rangle\right| \chi_{Q}\right)^{s_{2}}\right]^{1 / s_{2}} \|_{L^{q}} \\
& =C\left\|\left\{\left\langle f, \phi_{Q}^{1}\right\rangle\right\}\right\|_{\dot{f}_{p}^{\alpha, s_{1}}}\left\|\left\{\left\langle g, \phi_{Q}^{2}\right\rangle\right\}\right\|_{\dot{f}_{q}^{\beta, s_{2}}} \leq C\|f\|_{\dot{F}_{p}^{\alpha, s_{1}}}\|g\|_{\dot{F}_{q}^{\beta, s_{2}}},
\end{aligned}
$$

where we used (14), since $\left\{\phi_{Q}^{1}\right\}_{Q \in \mathcal{D}}$ and $\left\{\phi_{Q}^{2}\right\}_{Q \in \mathcal{D}}$ are families of dual molecules for $\dot{F}_{p}^{\alpha, s_{1}}$ and $\dot{F}_{q}^{\beta, s_{2}}$ respectively (see Remark 2.2). 
Á. Bényi, D. Maldonado, A. Nahmod, and R. Torres: Bilinear Paraproducts Revisited

\section{References}

[1] Á. Bényi, Bilinear singular integrals and pseudodifferential operators, Ph.D. Thesis, University of Kansas, 2002.

[2] Á. Bényi, Bilinear singular integral operators, smooth atoms and molecules, J. Fourier Anal. Appl. 9 no. 3 (2003), 301-319.

[3] Á. Bényi and R. H. Torres, Symbolic calculus and the transposes of bilinear pseudodifferential operators, Comm. P.D.E. 28 (2003), 1161-1181.

[4] Á. Bényi and N. Tzirakis, Multilinear almost diagonal estimates and applications, Studia Math. 164.1 (2004), 75-89.

[5] J.M. Bony, Calcul symbolique et propagation des singularités pour les équations aux dérivées partielles non-linéaires, Annales Scientifiques de l'École Normale Supérieure Sér. 4, 14 no. 2 (1981), 209-246.

[6] M. Christ and J.-L. Journé, Polynomial growth estimates for multilinear singular integral operators, Acta Math. 159 (1987), 51-80.

[7] R. R. Coifman, S. Dobyinsky, and Y. Meyer, Opérateurs bilinéaires et renormalization, in Essays on Fourier Analysis in Honor of Elias M. Stein, C. Fefferman, R. Fefferman, S. Wainger (eds), Princeton University Press, Princeton NJ, 1995.

[8] R. R. Coifman and Y. Meyer, Au-delà des opérateurs pseudo-différentiels, Astèrisque 57, 1978.

[9] R. R. Coifman and Y. Meyer Wavelets: Calderón-Zygmund and multilinear operators, Cambridge Univ. Press, Cambridge, United Kingdom, 1997.

[10] G. David and J-L. Journé, A boundedness criterion for generalized Calderón-Zygmund operators, Ann. of Math. 120 (1984), 371-397.

[11] M. Frazier, B. Jawerth, A discrete transform and decompositions of distribution spaces, J. Func. Anal. 93 (1990), $34-$ 169.

[12] M. Frazier, B. Jawerth, and G. Weiss, Littlewood-Paley theory and the study of function spaces, CBMS Regional Conference Series in Mathematics 79, 1991.

[13] J. Gilbert and A. Nahmod, Bilinear operators with non-smooth symbols. I, J. Fourier Anal. Appl. 5 (2001), $435-467$.

[14] J. Gilbert and A. Nahmod, $L^{p}$-boundedness of time-frequency paraproducts. II, J. Fourier Anal. Appl. 8 (2002), 109-172.

[15] L. Grafakos and N. Kalton, Multilinear Calderon-Zygmund operators on Hardy spaces, Collectanea Mathematica 52 (2001), 169-179.

[16] L. Grafakos and R. H. Torres, Discrete decompositions for bilinear operators and almost diagonal conditions, Trans. Amer. Math. Soc. 354 (2002), 1153-1176.

[17] L. Grafakos and R. H. Torres, Multilinear Calderón-Zygmund theory, Adv. in Math. 165 (2002), 124-164.

[18] L. Grafakos and R. H. Torres, Maximal operator and weighted norm inequalities for multilinear singular integrals, Indiana Univ. Math. J. 51 No. 5 (2002), 1261-1276.

[19] C. Kenig and E. Stein, Multilinear estimates and fractional integration, Math. Res. Lett. 6 (1999), 1-15. Erratum in Math. Res. Lett. 6 (1999), no. 3-4, 467.

[20] M. Lacey and J. Metcalfe, Paraproducts in one and several parameters, to appear in Forum Math.

[21] M. Lacey and C. Thiele, $L^{p}$ estimates on the bilinear Hilbert transform for $2<p<\infty$, Ann. of Math. 146 (1997), 693-724.

[22] M. Lacey and C. Thiele, On Calderón's conjecture, Ann. of Math. 149 (1999), 475-496.

[23] Y. Meyer, Ondelettes et opérateurs I and II, Hermann Ed., Paris, 1990.

[24] C. Muscalu, J. Pipher, T. Tao, and C. Thiele, Bi-parameter paraproducts, Acta Math. 193, (2004), 269-296.

[25] C. Muscalu, J. Pipher, T. Tao, and C. Thiele, Multi-parameter paraproducts, Rev. Mat. Iberoamericana 22 (2006), 963-976.

[26] C. Muscalu, T. Tao, and C. Thiele, Multilinear operators given by singular multipliers, J. Amer. Math. Soc. 15 (2002), 469-496.

[27] J. Peetre, New thoughts on Besov spaces, Durham, NC: Duke University Press, 1976.

[28] C. Thiele, Wave Packet Analysis, CBMS Regional Conference Series in Mathematics 105, 2006.

[29] R. H. Torres, Boundedness results for operators with singular kernels on distribution spaces, Mem. Amer. Math. Soc. 442, 1991.

[30] H. Triebel, Multiplication properties of the spaces $B_{p, q}^{s}$ and $F_{p, q}^{s}$. Quasi-Banach algebras of functions, Ann. Mat. Pura Appl. (4) 113 (1977), 33-42.

[31] H. Triebel, Multiplication properties of Besov spaces, Ann. Mat. Pura Appl. (4) 114 (1977), 87-102.

[32] H. Triebel, Theory of function spaces, Monographs in Mathematics, Vol. 78, Birkhauser Verlag, Basel, 1983.

[33] K. Wang, The generalization of paraproducts and the full T1 theorem for Sobolev and Triebel-Lizorkin spaces, J. Math. Anal. Appl. 2092 (1997), 317-340.

[34] K. Wang, The full T1 theorem for certain Triebel-Lizorkin spaces, Math. Nachr. 197 (1999), 103-133.

[35] A. Youssif, Bilinear operators and the Jacobian-determinant on Besov spaces, Indiana Univ. Math. J. 45 (1996), 381396. 